\title{
Evidence for Top Quark Production in Nucleus-Nucleus Collisions
}

\author{
A. M. Sirunyan et al. \\ (CMS Collaboration)
}

(Received 28 July 2020; accepted 7 October 2020; published 24 November 2020)

\begin{abstract}
Ultrarelativistic heavy ion collisions recreate in the laboratory the thermodynamical conditions prevailing in the early universe up to $10^{-6} \mathrm{sec}$, thereby allowing the study of the quark-gluon plasma (QGP), a state of quantum chromodynamics (QCD) matter with deconfined partons. The top quark, the heaviest elementary particle known, is accessible in nucleus-nucleus collisions at the CERN LHC, and constitutes a novel probe of the QGP. Here, we report the first evidence for the production of top quarks in nucleus-nucleus collisions, using lead-lead collision data at a nucleon-nucleon center-of-mass energy of $5.02 \mathrm{TeV}$ recorded by the CMS experiment. Two methods are used to measure the cross section for top quark pair production $\left(\sigma_{t \bar{t}}\right)$ via the selection of charged leptons (electrons or muons) and bottom quarks. One method relies on the leptonic information alone, and the second one exploits, in addition, the presence of bottom quarks. The measured cross sections, $\sigma_{t \bar{t}}=2.54_{-0.74}^{+0.84}$ and $2.03_{-0.64}^{+0.71} \mu \mathrm{b}$, respectively, are compatible with expectations from scaled proton-proton data and QCD predictions.
\end{abstract}

DOI: 10.1103/PhysRevLett.125.222001

Since the top quark discovery at the Fermilab Tevatron more than twenty years ago [1,2], top quarks have been measured at the LHC in proton-proton $(p p)[3-7]$ as well as proton-nucleus [8] collisions, but so far have not been observed in nucleus-nucleus collisions because of insufficient nucleon-nucleon (NN) center-of-mass energies $\left(\sqrt{s_{\mathrm{NN}}}\right)$ or integrated luminosities. The multi-TeV energies available at the CERN LHC have opened up the possibility to measure, for the first time, the top quark in lead-lead $(\mathrm{PbPb})$ collisions [9]. More specifically, the top quark constitutes a novel and theoretically precise probe of the nuclear parton distribution functions (nuclear PDFs, or $n P D F s$, in the poorly explored region where partons have a large fraction of the nucleon momentum, as well as of the properties of the produced quark-gluon plasma (QGP) $[9,10]$. First, precise knowledge of nPDFs is a key prerequisite to extract detailed information on the QGP properties from the experimental data. Second, top quarks, on average, decay on a timescale similar to the formation of the QGP, hence offering a unique opportunity to study its time evolution [10]. The study presented here shows evidence for the production of the top quark in $\mathrm{PbPb}$ collisions at $\sqrt{s_{\mathrm{NN}}}=5.02 \mathrm{TeV}$ with an integrated luminosity of $(1.7 \pm 0.1) \mathrm{nb}^{-1}[11]$ as recorded by the CMS detector [12].

*Full author list given at the end of the article.

Published by the American Physical Society under the terms of the Creative Commons Attribution 4.0 International license. Further distribution of this work must maintain attribution to the author(s) and the published article's title, journal citation, and DOI. Funded by SCOAP ${ }^{3}$.
The top quark - the heaviest elementary particle known-is produced at hadron colliders predominantly in pairs $(t \bar{t})$ through quantum chromodynamics (QCD) processes, mostly gluon-gluon fusion at the LHC, and is thereby a sensitive probe of the gluon PDF of the incoming nucleons [13]. Once produced, it decays with almost $100 \%$ probability into a $W$ boson and a bottom $(b)$ quark. Top quark pair production is thereby characterized by final states comprising the decay products of the two $W$ bosons, and two $b$ jets, resulting from the hadronization products of $b$ quarks. The dilepton final states, in which both $W$ bosons decay into electrons $(e)$ or muons $(\mu)$ and the corresponding neutrinos $(\nu)$, are the cleanest final states for the $t \bar{t}$ signal measurement, despite their relatively small branching fraction $\mathcal{B}\left(t \bar{t} \rightarrow \ell^{+} \ell^{-} \nu_{\ell} \nu_{\ell} b \bar{b}\right)=5.25 \%$ [14], with $\ell^{ \pm}=e^{ \pm}$, $\mu^{ \pm}$. See the Supplemental Material [15] for a candidate $t \bar{t}$ event.

In this Letter, the measurement of the $t \bar{t}$ cross section $\left(\sigma_{t \bar{t}}\right)$ in three dilepton final states, i.e., $e^{+} e^{-}, \mu^{+} \mu^{-}$, and $e^{ \pm} \mu^{\mp}$, is performed by (i) making use of the final-state dilepton kinematic properties alone, and (ii) imposing extra requirements on the number of jets "tagged" as originating from $b$ quarks (referred to as " $b$-tagged jets"). The first method is motivated by the fact that leptons propagate unscathed through the QGP, thereby providing favorable conditions for the detection of $t \bar{t}$ production. The second method, which enhances the signal over background in standard $p p$ analyses, is applied with realistic estimates of the impact of $b$ quark energy loss, also known as "jet quenching," in the QGP [16].

The main feature of the CMS detector is a superconducting solenoid, providing a magnetic field of $3.8 \mathrm{~T}$. Within the solenoid volume is a silicon pixel and 
strip tracker, which is used to detect charged particles, a lead tungstate crystal electromagnetic calorimeter, and a brass and scintillator hadron calorimeter. Hadron forward calorimeters extend the pseudorapidity coverage up to $|\eta|=5.2$. Muons are detected in gas-ionization chambers embedded in the steel flux-return yoke outside the solenoid. A more detailed description of the CMS detector, together with a definition of the coordinate system, the relevant kinematic variables (e.g., the transverse momentum $p_{T}$ ), and the physics-object reconstruction, can be found in Ref. [12]. The data sample is collected with a two-level trigger system [17]: at level 1 events are selected by custom hardware processors while the high-level trigger uses fast versions of the off-line software. All particle candidates are reconstructed with the particle-flow algorithm [18] using an optimized combination of information from the various elements of the CMS detector.

The data sample is filtered to favor events with two opposite-sign (OS) high- $p_{T}$ leptons, with $p_{T}>$ 25(20) GeV and $|\eta|<2.1(2.4)$ for the electron [19] (muon [20]) candidates, that do not belong to jets reconstructed using a distance parameter of $0.4[21,22]$ and are thus isolated from nearby hadronic activity. The correction for the underlying event (UE) formed from soft processes is done on a particle-by-particle basis using an estimation of the median of the energy density $\rho$ [21-23] in the event for the lepton isolation, and the "constituent subtraction" method $[24,25]$ for the jet constituents. The characteristic additional presence of two $b$-tagged jets in the $t \bar{t}$ decay chain is then used, in our second method only, to enhance the sensitivity to the top quark signal. Jets must have $p_{T}>$ $30 \mathrm{GeV}$ and $|\eta|<2.0$, and are considered as $b$ tagged if an optimized "combined secondary vertex" (CSV) discriminator [26] produces a value above a certain threshold for the probability of the jet to stem from the hadronization of the $b$ quark. The $b$ tagging efficiency depends on the geometric overlap of the two colliding nuclei as given by the centrality percentile [27]. This percentile is defined from percentages corresponding to fractions of the total inelastic hadronic cross section, e.g., with $0 \%$ denoting the full overlap of the two colliding nuclei. After the selection criteria, the $b$ tagging efficiency in a NN $\rightarrow t \bar{t}$ Monte Carlo (MC) simulation sample, generated at next-to-leading order (NLO) in QCD using the MadGraph5_aMC@NLO (v2.4.2) [28] program and interfaced to the "tuned" [29] PYTHIA8 (v2.3.0) [30] MC event generator, is approximately $60(70) \%$, with a misidentification rate of 5 (2)\%, in the 0-30 (30-100)\% centrality interval. The two jets with the highest CSV discriminator values are used to count the $b$-tagged jet multiplicity, $N_{b \text {-tag }}$, and classify the selected events into the " $0 b "\left(N_{b \text {-tag }}=0\right), " 1 b "\left(N_{b \text {-tag }}=1\right)$, and " $2 b "\left(N_{b \text {-tag }}=2\right)$ jet categories.

The main background is Drell-Yan quark-antiquark annihilation into lepton-antilepton pairs through $Z$ bosons or virtual photons (a process referred to as " $Z / \gamma^{*}$ "). It contaminates all final states with either off shell (in $e^{+} e^{-}$ and $\mu^{+} \mu^{-}$) or $Z / \gamma^{*} \rightarrow \tau^{+} \tau^{-} \rightarrow e^{ \pm} \mu^{\mp}+X \quad\left(\right.$ in $e^{ \pm} \mu^{\mp}$ ) decays, where " $X$ " represents other particles. The $Z / \gamma^{*}$ process is modeled at NLO using the MadGraph5_aMC@NLO simulation with corrections obtained from data, as detailed below. In the $e^{ \pm} \mu^{\mp}$ final state, in particular, there are additional contaminations from $W$ boson production in association with jets (" $W+$ jets"), $Z / \gamma^{*}$ with one unreconstructed lepton, and QCD multijet events. In these cases, the produced jets are mainly from heavy quarks eventually decaying into high- $p_{T}$ leptons that are erroneously identified as being isolated. These latter processes, referred to in what follows as "nonprompt" background, are directly derived from control regions in the data, as explained next. Smaller background contributions from single top quark and $W$ boson (" $t W$ "), and $W W, W Z$, and $Z Z$ (collectively referred to as " $V V$ ") production, are directly estimated from MC simulation with POWHEG [31,32]. In all simulated samples, the EPPS16 NLO nPDF [33], with CT14 NLO free-nucleon PDF [34], the strong coupling constant at the $Z$ boson mass $\alpha_{\mathrm{S}}\left(m_{Z}\right)=0.118 \pm 0.001$ [14], and the top quark mass $m_{t}=172.5 \mathrm{GeV}$ [35] are used as input. At the step of detector digitization, each hard scattering event is placed at the same primary-vertex [36] location as a heavy ion background event generated with HYDJET (v1.9) [37], to mimic the effects of the UE without any QGP-induced modifications of the final-state particles from the top quark decay. Finally, all simulated samples include an emulation of the full CMS detector response, based on GEANT4 [38], and a realistic description of the luminous region produced by the collisions.

The $Z / \gamma^{*}$ simulation provides a good modeling of the dilepton kinematic properties, except for the low- $p_{T}$ region where multiple soft-gluon emission dominates and the agreement is slightly worse. We thus apply correction ("scale") factors to the MC simulation using events in data enriched with $Z / \gamma^{*} \rightarrow \ell^{+} \ell^{-}$candidates. The scale factors are measured as a function of centrality, but no particular centrality dependence is seen. The difference between the corrected and uncorrected $\mathrm{MC}$ distributions is considered as the $Z / \gamma^{*} p_{T}$ modeling uncertainty. Events in the $e^{+} e^{-}$and $\mu^{+} \mu^{-}$final states with dilepton invariant mass $m\left(\ell^{+} \ell^{-}\right)$in the proximity of the $Z$ boson mass $m_{Z}$ [14] $\left[76<m\left(\ell^{+} \ell^{-}\right)<106 \mathrm{GeV}\right]$ are rejected, and their number is used to control the normalization of the corrected MC distributions outside the $m_{Z}$ region. The overall normalization of the nonprompt background is estimated by forming a "same-sign" (SS) control region, i.e., applying the same criteria as to the signal selection, but requiring SS lepton pairs. The SS dilepton events predominantly contain at least one misidentified lepton. The scaling from the SS control to the signal regions is performed assuming the ratio of the number of OS to SS events containing misidentified leptons to be unity. To estimate the distribution of the nonprompt background, an event mixing technique is developed. The mixing is performed for each lepton in a pool of 100 different events sharing the same 
features (i.e., lepton charge and flavor, and whether originating from on-shell or off-shell $Z$ bosons). Each lepton is randomly substituted, and the kinematic variables are recomputed with this new dilepton hypothesis. A multidimensional distance is calculated with respect to the original event using a nearest-neighbor algorithm [39]. The variables entering the algorithm are the centrality, $\rho$, lepton $p_{T}$ and isolation, and the magnitude of the $p_{T}$ of the dilepton system ("dilepton $p_{T}$ "). The highest ranked mixed events, corresponding to the smallest multidimensional distance, are chosen as the nominal distribution. Differences with respect to the distributions obtained using events further apart in this multidimensional distance, i.e., lower ranked hypotheses, are considered as a source of systematic uncertainty.

For both the dilepton-only and dilepton plus $b$-tagged jets methods, a boosted decision tree (BDT) [40] classifier is trained on the simulated $t \bar{t}$ signal versus the overall $Z / \gamma^{*} \rightarrow e^{+} e^{-}, \mu^{+} \mu^{-}$background. This classifier is based exclusively on leptonic quantities to minimize effects from the imprecise knowledge of the jet properties in the heavy ion environment. The BDT exploits the properties of the leading- and subleading- $p_{T}$ leptons, denoted by " $\ell_{1}$ " and " $\ell_{2}$," respectively, and their correlations. As input to the BDT classifier, the following variables are used in descending order of importance: (i) the $p_{T}$ of the leading lepton $p_{T}\left(\ell_{1}\right)$; (ii) the normalized momentum imbalance between $\ell_{1}$ and $\ell_{2}, A_{p_{T}}=\left[p_{T}\left(\ell_{1}\right)-p_{T}\left(\ell_{2}\right)\right] /\left[p_{T}\left(\ell_{1}\right)+p_{T}\left(\ell_{2}\right)\right]$; (iii) the dilepton $p_{T}$; (iv) the absolute pseudorapidity of the dilepton system; (v) the absolute azimuthal separation between $\ell_{1}$ and $\ell_{2}$; and (vi) the sum of the absolute $\eta$ of $\ell_{1}$ and $\ell_{2}$.

Figure 1 (left) shows the observed BDT discriminator distribution for the dilepton-only method in the higher sensitivity $e^{ \pm} \mu^{\mp}$ final state (see Supplemental Material [15] for the $e^{+} e^{-}$and $\mu^{+} \mu^{-}$final states). The $t \bar{t}$ signal and various sources of background are also shown, indicated as "prefit expected" as they are not adjusted according to any statistical treatment ("fit"). The $Z / \gamma^{*}$ background is normalized to the next-to-next-to-leading order (NNLO) cross section from the FEWZ (v3.1.rc) program [41], and the $V V$ and $t W$ contributions are normalized to the NLO and the approximate NNLO cross sections calculated with MCFM (v8.0) [42] and from Ref. [43], respectively. The classifier separates well the $t \bar{t}$ signal from the $Z / \gamma^{*}$ background in all final states. The $t \bar{t}$ signal (red histogram) populates the high-BDT discriminator values. The uncertainties in the data are statistical only, while the uncertainties in the backgrounds include a prefit expectation of the systematic uncertainty.

Profile likelihood fits $[44,45]$ to binned BDT discriminator distributions are performed separately for the dilepton-only and dilepton plus $b$-tagged jets methods. The best fit values of "signal strength" $(\mu)$, their uncertainty $\Delta \mu$ (corresponding to a $68 \%$ confidence level), and the
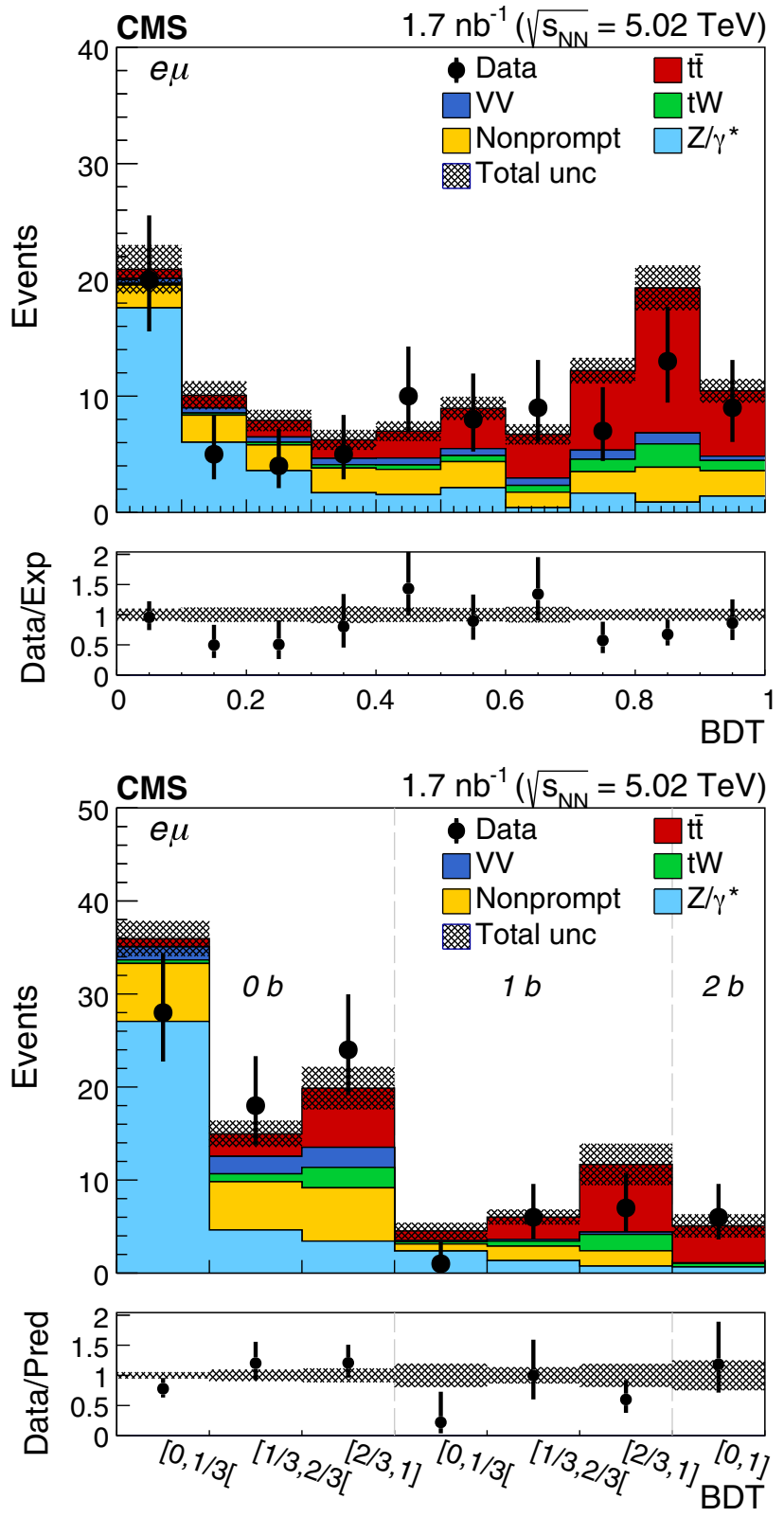

FIG. 1. Observed and prefit expected (left) or postfit predicted (right) BDT discriminator distributions in the $e^{ \pm} \mu^{\mp}$ final state either inclusively (left) or separately in the $0 b-, 1 b$-, and $2 b$ tagged jet multiplicity categories (right). The data are shown with markers, and the signal and background processes with filled histograms. The vertical bars on the markers represent the statistical uncertainties in data. The hatched regions show the uncertainties in the sum of $t \bar{t}$ signal and backgrounds. The lower panels display the ratio of the data to predictions, including the $t \bar{t}$ signal, with bands representing the uncertainties in the predictions.

significance (in units of standard deviations) of the $t \bar{t}$ process against the background-only hypothesis, are obtained following the procedure described in Sec. 3.2 of Ref. [46] and the frequentist paradigm using the profile likelihood ratio as a test statistic [47], accordingly. 
The value of $\mu$ is defined as the ratio of the observed $\sigma_{\bar{t} \bar{t}}$ to the expectation from theory, i.e., $\mu=\sigma_{t \bar{t}} / \sigma_{\bar{t} \bar{t}}^{\text {th }}$. The theoretical cross section $\sigma_{t \bar{t}}^{\mathrm{th}}=\sigma_{\mathrm{PbPb} \rightarrow \bar{t}+X}^{\mathrm{NNLO}+\mathrm{NNLL}}=$ $3.22_{-0.35}^{+0.38}(\mathrm{nPDF} \oplus \mathrm{PDF})_{-0.10}^{+0.09}$ (scale) $\mu \mathrm{b}$, calculated with the TOP++ (v2.0) program [48,49] at NNLO in QCD, including soft-gluon resummation at next-to-next-toleading logarithmic (NNLL) accuracy, with the nuclear EPPS16 [33] and free-nucleon CT14 [34] PDFs. The same calculation but with the free-nucleon CT14 and NNPDF30 [50] NNLO PDFs (scaled by the square of the number of nucleons in the $\mathrm{Pb}$ nucleus, $A^{2}=208^{2}$ ) yields $\quad \sigma_{\mathrm{PbPb} \rightarrow \bar{t}+X}^{\mathrm{NNLO}+\mathrm{NNL}}=3.04_{-0.14}^{+0.18}(\mathrm{PDF})_{-0.10}^{+0.08}($ scale $) \quad$ and $2.98 \pm 0.14\left[\mathrm{PDF} \oplus \alpha_{\mathrm{S}}\left(m_{Z}\right)\right]_{-0.10}^{+0.08}$ (scale) $\mu \mathrm{b}$, respectively. The small difference between the cross sections obtained with nuclear and free-nucleon PDFs arises from the nPDF "antishadowing" effect, which is only mildly dependent on $\sqrt{s_{\mathrm{NN}}}[9]$.

In the dilepton-only method, the extracted values of $\mu=$ $0.79_{-0.23}^{+0.26}$ (where contributions to $\Delta \mu$ are statistical and systematic in nature) and significance of 3.8 standard deviations constitute the first evidence of $t \bar{t}$ production in nucleusnucleus collisions. As can be seen in the prefit distribution of Fig. 1 (left), the data are somewhat below the expectation at the high-BDT discriminator values. This is also reflected in differences in $\mu$ and significance, where the expected values are $\mu=1.00_{-0.23}^{+0.25}$ and 4.8 standard deviations, respectively.

Events in which $N_{b \text {-tag }} \geq 1$ are expected to be very pure in the $t \bar{t}$ signal process. Since $b$ quarks are affected by finalstate energy loss in the QGP, we take into account the centrality-dependent impact from jet quenching on $N_{b \text {-tag }}$. We make use of a jet quenching model [51,52] that is consistent with the CMS $b$ jet data [16], estimating the expected migration of $t \bar{t}$ signal events among the $0 b-$, $1 b$-, or $2 b$-tagged jet categories. A combined profile likelihood fit, introducing a parameter $\varepsilon_{b}$ that correlates the number of $t \bar{t}$ signal events in the three $b$-tagged jet categories based on multinomial probabilities [5], is thus expected to control better the background contamination. We include in the likelihood the effects on $\varepsilon_{b}$ from jet quenching (comparing the maximum with no $b$ quark energy loss scenarios), and the intrinsic uncertainties in the $b$ tagging efficiency and misidentification rate. The values of the observed (expected) signal strength and significance are $\mu=0.63_{-0.20}^{+0.22}\left(1.00_{-0.21}^{+0.23}\right)$ and $4.0(5.8)$ standard deviations, respectively. Figure 1 (right) compares the data to the $t \bar{t}$ signal and various sources of background adjusted according to the fit procedure ("postfit predicted") for the dilepton plus b-tagged jets method in the $e^{ \pm} \mu^{\mp}$ final state (see Supplementl Material [15] for the $e^{+} e^{-}$and and $\mu^{+} \mu^{-}$final states). The BDT distribution for the $Z / \gamma^{*}$ background is taken from the MC simulation, after scaling the event yield in each $N_{b \text {-tag }}$ bin to the corresponding $N_{b \text {-tag }}$ distribution observed in data within the $m_{Z}$ region.
Sources of experimental uncertainties, incorporated into the likelihoods via "nuisance parameters," include the lepton selection efficiency found using a "tag-and-probe" method [53] (6\%), integrated luminosity [11] (5\%), and the normalization of the background based on control samples in data (12\%). The statistical uncertainties in the $t \bar{t}$ signal and background distributions (7\%) are estimated separately. The dilepton plus b-tagged jets method is, in addition, affected by the uncertainty in $\varepsilon_{b}(6 \%)$, and the jet energy scale and resolution (2\%) estimated following the methodology of Ref. [54]. Sources of theoretical uncertainty included in the likelihoods are the nPDF parametrization (derived from the $54+40$ eigenvalues of the EPPS16 + CT14 sets), the choice of renormalization and factorization scales (within a factor of two from their default values of $\left.\mu_{R}=\mu_{F}=m_{t}\right)$, and $\alpha_{S}\left(m_{Z}\right)$ are included $(<1 \%)$. We also take into account the uncertainties in the $p_{T}$ modeling of the $t \bar{t}$ signal and $Z / \gamma^{*}$ background distributions $(5 \%)$ as well as in the top quark mass $(<1 \%)$. The precision of the two methods is dominated by the statistical uncertainty $(\approx 28 \%)$.

The inclusive $t \bar{t}$ production cross sections (for the dilepton-only and dilepton plus $b$-tagged jets methods) are finally obtained in the combined $e^{+} e^{-}, \mu^{+} \mu^{-}$, and $e^{ \pm} \mu^{\mp}$ final states multiplying the best fit $\mu$ values of $0.79_{-0.23}^{+0.26}$ and $0.63_{-0.20}^{+0.22}$ by the theoretical expectation. We measure $\sigma_{t \bar{t}}=$ $2.54_{-0.74}^{+0.84}$ and $2.03_{-0.64}^{+0.71} \mu \mathrm{b}$ for the two methods, i.e., smaller than, but still consistent with, the theoretical predictions at

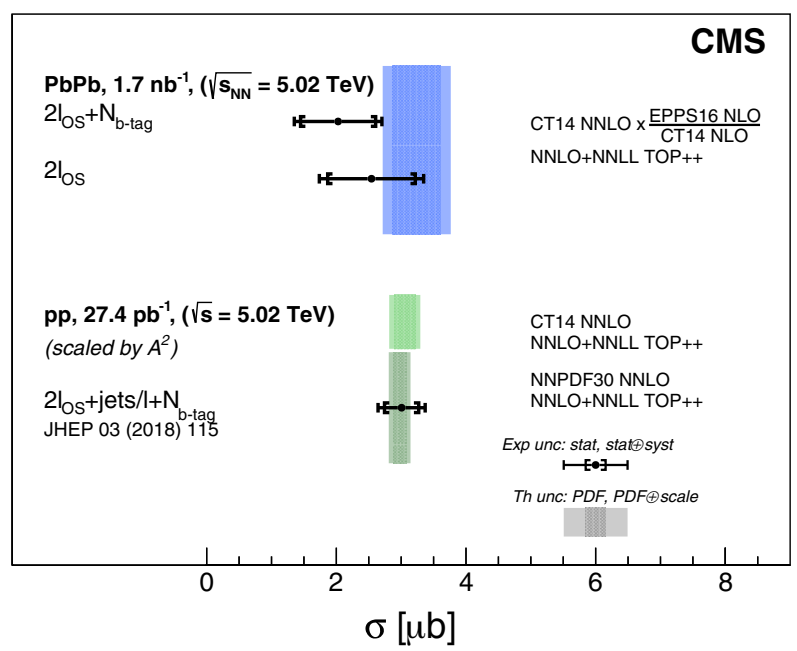

FIG. 2. Inclusive $t \bar{t}$ cross sections measured with two methods in the combined $e^{+} e^{-}, \mu^{+} \mu^{-}$, and $e^{ \pm} \mu^{\mp}$ final states in $\mathrm{PbPb}$ collisions at $\sqrt{s_{\mathrm{NN}}}=5.02 \mathrm{TeV}$, and $p p$ results at $\sqrt{s}=5.02 \mathrm{TeV}$ (scaled by $A^{2}$ ) from Ref. [6]. The measurements are compared with theoretical predictions at NNLO + NNLL accuracy in QCD $[48,49]$. The inner (outer) experimental uncertainty bars include statistical (statistical and systematic, added in quadrature) uncertainties. The inner (outer) theoretical uncertainty bands correspond to nuclear $[33,55]$ or free-nucleon $[34,50] \mathrm{PDF}$ (PDF and scale, added in quadrature) uncertainties. 
NNLO + NNLL accuracy in QCD. Despite the expected antishadowing effect, the data appear below the theoretical expectations with or without nPDF effects. Figure 2 presents a summary of the extracted cross sections, including the measurement in $p p$ collisions at $\sqrt{s}=5.02 \mathrm{TeV}$ [6] scaled by $A^{2}$, compared with the corresponding theoretical predictions.

In summary, evidence for top quark pair $(t \bar{t})$ production is presented for the first time in nucleus-nucleus collisions, irrespective of any possible final-state interactions of the studied top quark decay products (charged leptons and bottom quarks) with the quark-gluon plasma (QGP). Using lead-lead collisions with a total integrated luminosity of $(1.7 \pm 0.1) \mathrm{nb}^{-1}$ at a nucleon-nucleon center-ofmass energy of $5.02 \mathrm{TeV}$, we measure the inclusive $t \bar{t}$ cross section $\left(\sigma_{t \bar{t}}\right)$ utilizing the leptons only, and in a second method, in addition, the bottom quarks. The extracted $\sigma_{t \bar{t}}=2.54_{-0.74}^{+0.84}$ and $2.03_{-0.64}^{+0.71} \mu \mathrm{b}$ in the two methods, respectively, are compatible with, though somewhat lower than, the expectations from scaled protonproton data and perturbative quantum chromodynamics calculations. This measurement is just the first step in using the top quark as a novel and powerful probe of the QGP.

We congratulate our colleagues in the CERN accelerator departments for the excellent performance of the LHC and thank the technical and administrative staffs at CERN and at other CMS institutes for their contributions to the success of the CMS effort. In addition, we gratefully acknowledge the computing centers and personnel of the Worldwide LHC Computing Grid for delivering so effectively the computing infrastructure essential to our analyses. Finally, we acknowledge the enduring support for the construction and operation of the LHC and the CMS detector provided by the following funding agencies: BMBWF and FWF (Austria); FNRS and FWO (Belgium); CNPq, CAPES, FAPERJ, FAPERGS, and FAPESP (Brazil); MES (Bulgaria); CERN; CAS, MoST, and NSFC (China); COLCIENCIAS (Colombia); MSES and CSF (Croatia); RIF (Cyprus); SENESCYT (Ecuador); MoER, ERC IUT, PUT and ERDF (Estonia); Academy of Finland, MEC, and HIP (Finland); CEA and CNRS/IN2P3 (France); BMBF, DFG, and HGF (Germany); GSRT (Greece); NKFIA (Hungary); DAE and DST (India); IPM (Iran); SFI (Ireland); INFN (Italy); MSIP and NRF (Republic of Korea); MES (Latvia); LAS (Lithuania); MOE and UM (Malaysia); BUAP, CINVESTAV, CONACYT, LNS, SEP, and UASLP-FAI (Mexico); MOS (Montenegro); MBIE (New Zealand); PAEC (Pakistan); MSHE and NSC (Poland); FCT (Portugal); JINR (Dubna); MON, RosAtom, RAS, RFBR, and NRC KI (Russia); MESTD (Serbia); SEIDI, CPAN, PCTI, and FEDER (Spain); MOSTR (Sri Lanka); Swiss Funding Agencies (Switzerland); MST (Taipei); ThEPCenter, IPST, STAR, and NSTDA (Thailand); TUBITAK and TAEK (Turkey); NASU (Ukraine); STFC (United Kingdom); DOE and NSF (USA).

[1] F. Abe et al. (CDF Collaboration), Observation of Top Quark Production in $p \bar{p}$ Collisions, Phys. Rev. Lett. 74, 2626 (1995).

[2] S. Abachi et al. (D0 Collaboration), Observation of the Top Quark, Phys. Rev. Lett. 74, 2632 (1995).

[3] ATLAS Collaboration, Measurement of the $t \bar{t}$ production cross section and lepton differential distributions in $e \mu$ dilepton events from $p p$ collisions at $\sqrt{s}=13 \mathrm{TeV}$ with the ATLAS detector, Eur. Phys. J. C 80, 528 (2020).

[4] LHCb Collaboration, Measurement of forward top pair production in the dilepton channel in $\mathrm{pp}$ collisions at $\sqrt{s}=13 \mathrm{TeV}$, J. High Energy Phys. 08 (2018) 174.

[5] CMS Collaboration, Measurement of the $t \bar{t}$ production cross section, the top quark mass, and the strong coupling constant using dilepton events in pp collisions at $\sqrt{s}=13 \mathrm{TeV}$, Eur. Phys. J. C 79, 368 (2019).

[6] CMS Collaboration, Measurement of the inclusive $t \bar{t}$ cross section in pp collisions at $\sqrt{s}=5.02 \mathrm{TeV}$ using final states with at least one charged lepton, J. High Energy Phys. 03 (2018) 115.

[7] CMS Collaboration, Measurement of the $t \bar{t}$ production cross section in the all-jets final state in pp collisions at $\sqrt{s}=8 \mathrm{TeV}$, Eur. Phys. J. C 76, 128 (2016).

[8] CMS Collaboration, Observation of Top Quark Production in Proton-Nucleus Collisions, Phys. Rev. Lett. 119, 242001 (2017).

[9] D. d'Enterria, K. Krajczár, and H. Paukkunen, Top quark production in proton-nucleus and nucleus-nucleus collisions at LHC energies and beyond, Phys. Lett. B 746, 64 (2015).

[10] L. Apolinário, J. G. Milhano, G. P. Salam, and C. A. Salgado, Probing the Time Structure of the Quark-Gluon Plasma with Top Quarks, Phys. Rev. Lett. 120, 232301 (2018).

[11] CMS Collaboration, CMS luminosity measurement using 2016 proton-nucleus collisions at nucleon-nucleon centerof-mass energy of $8.16 \mathrm{TeV}$, CMS Physics Analysis Summary, CERN Report No. CMS-PAS-LUM-17-002, 2018, http://cds.cern.ch/record/2628652.

[12] CMS Collaboration, The CMS experiment at the CERN LHC, J. Instrum. 3, S08004 (2008).

[13] M. Czakon, M. L. Mangano, A. Mitov, and J. Rojo, Constraints on the gluon PDF from top quark pair production at hadron colliders, J. High Energy Phys. 07 (2013) 167.

[14] M. Tanabashi et al. (Particle Data Group), Review of particle physics, Phys. Rev. D 98, 030001 (2018).

[15] See Supplemental Material http://link.aps.org/supplemental/ 10.1103/PhysRevLett.125.222001 for $t \bar{t}$ event display and BDT distributions in the $e^{+} e^{-}$and $\mu^{+} \mu^{-}$final states.

[16] CMS Collaboration, Evidence of $b$ Jet Quenching in $\mathrm{PbPb}$ Collisions at $\sqrt{s_{\mathrm{NN}}}=2.76 \mathrm{TeV}$, Phys. Rev. Lett. 113, 132301 (2014); Erratum, Phys. Rev. Lett. 115, 029903 (2015). 
[17] CMS Collaboration, The CMS trigger system, J. Instrum. 12, P01020 (2017).

[18] CMS Collaboration, Particle-flow reconstruction and global-event description with the CMS detector, J. Instrum. 12, P10003 (2017).

[19] CMS Collaboration, Performance of electron reconstruction and selection with the CMS detector in proton-proton collisions at $\sqrt{s}=8 \mathrm{TeV}$, J. Instrum. 10, P06005 (2015).

[20] CMS Collaboration, Performance of the CMS muon detector and muon reconstruction with proton-proton collisions at $\sqrt{s}=13 \mathrm{TeV}$, J. Instrum. 13, P06015 (2018).

[21] M. Cacciari, G. P. Salam, and G. Soyez, FastJet user manual, Eur. Phys. J. C 72, 1896 (2012).

[22] M. Cacciari, G. P. Salam, and G. Soyez, The anti- $k_{\mathrm{T}}$ jet clustering algorithm, J. High Energy Phys. 04 (2008) 063.

[23] M. Cacciari and G. P. Salam, Pileup subtraction using jet areas, Phys. Lett. B 659, 119 (2008).

[24] P. Berta, M. Spousta, D. W. Miller, and R. Leitner, Particlelevel pileup subtraction for jets and jet shapes, J. High Energy Phys. 06 (2014) 092.

[25] CMS Collaboration, Measurement of the Splitting Function in $p p$ and $\mathrm{PbPb}$ Collisions at $\sqrt{s_{\mathrm{NN}}}=5.02 \mathrm{TeV}$, Phys. Rev. Lett. 120, 142302 (2018).

[26] CMS Collaboration, Identification of heavy-flavor jets with the CMS detector in $p p$ collisions at $13 \mathrm{TeV}$, J. Instrum. 13, P05011 (2018).

[27] CMS Collaboration, Jet momentum dependence of jet quenching in $\mathrm{PbPb}$ collisions at $\sqrt{s_{\mathrm{NN}}}=2.76 \mathrm{TeV}$, Phys. Lett. B 712, 176 (2012).

[28] J. Alwall, R. Frederix, S. Frixione, V. Hirschi, F. Maltoni, O. Mattelaer, H. S. Shao, T. Stelzer, P. Torrielli, and M. Zaro, The automated computation of tree-level and next-to-leading order differential cross sections, and their matching to parton shower simulations, J. High Energy Phys. 07 (2014) 079.

[29] CMS Collaboration, Extraction and validation of a new set of CMS PYTHIA8 tunes from underlying-event measurements, Eur. Phys. J. C 80, 4 (2020).

[30] T. Sjöstrand, S. Ask, J. R. Christiansen, R. Corke, N. Desai, P. Ilten, S. Mrenna, S. Prestel, C. O. Rasmussen, and P. Skands, An introduction to PYTHIA 8.2, Comput. Phys. Commun. 191, 159 (2015).

[31] E. Re, Single-top $W t$-channel production matched with parton showers using the POWHEG method, Eur. Phys. J. C 71, 1547 (2011).

[32] P. Nason and G. Zanderighi, $W^{+} W^{-}, W Z$, and $Z Z$ production in the POWHEG-BOX-v2, Eur. Phys. J. C 74, 2702 (2014).

[33] K. J. Eskola, P. Paakkinen, H. Paukkunen, and C. A. Salgado, EPPS16: Nuclear parton distributions with LHC data, Eur. Phys. J. C 77, 163 (2017).

[34] S. Dulat, T.-J. Hou, J. Gao, M. Guzzi, J. Huston, P. Nadolsky, J. Pumplin, C. Schmidt, D. Stump, and C. P. Yuan, New parton distribution functions from a global analysis of quantum chromodynamics, Phys. Rev. D 93, 033006 (2016).

[35] CMS Collaboration, Measurement of the top quark mass in the all-jets final state at $\sqrt{s}=13 \mathrm{TeV}$ and combination with the lepton + jets channel, Eur. Phys. J. C 79, 313 (2019).

[36] CMS Collaboration, Technical proposal for the phase-II upgrade of the compact muon solenoid, CMS Technical proposal, CERN Reports No. CERN-LHCC-2015-010 and No. CMS-TDR-15-02, 2015, http://cds.cern.ch/record/ 2020886.

[37] I. P. Lokhtin and A. M. Snigirev, A model of jet quenching in ultrarelativistic heavy ion collisions and high- $p_{\mathrm{T}}$ hadron spectra at RHIC, Eur. Phys. J. C 45, 211 (2006).

[38] S. Agostinelli et al. (GEANT4 Collaboration), GEANT4-A simulation toolkit, Nucl. Instrum. Methods Phys. Res., Sect. A 506, 250 (2003).

[39] F. Pedregosa, G. Varoquaux, A. Gramfort, V. Michel, B. Thirion, O. Grisel, M. Blondel, P. Prettenhofer, R. Weiss, V. Dubourg, J. Vanderplas, A. Passos, D. Cournapeau, M. Brucher, M. Perrot, and E. Duchesnay, Scikit-learn: Machine learning in Python, J. Mach. Learn. Res. 12, 2825 (2011), http://jmlr.org/papers/v12/pedregosa11a.html.

[40] H. Voss, A. Höcker, J. Stelzer, and F. Tegenfeldt, TMVA, the toolkit for multivariate data analysis with ROOT, Proc. Sci., ACAT2007 (2007) 040.

[41] Y. Li and F. Petriello, Combining QCD and electroweak corrections to dilepton production in FEwz, Phys. Rev. D 86, 094034 (2012).

[42] J. M. Campbell, R. K. Ellis, and C. Williams, Vector boson pair production at the LHC, J. High Energy Phys. 07 (2011) 018 .

[43] N. Kidonakis, Top quark production, in Proceedings of the Helmholtz International Summer School on Physics of Heavy Quarks and Hadrons (HQ 2013) (Verlag Deutsches Elektronen-Synchrotron, Hamburg, 2014), p. 139, http://dx .doi.org/10.3204/DESY-PROC-2013-03/Kidonakis.

[44] W. Verkerke and D. P. Kirkby, The RooFit toolkit for data modelling, in Statistical Problems in Particle Physics, Astrophysics and Cosmology (PHYSTAT 05), edited by A. H. Jaffe (World Scientific, Oxford University, UK, 2006), http://dx.doi.org/10.1142/9781860948985_0052.

[45] L. Moneta, K. Belasco, K. S. Cranmer, S. Kreiss, A. Lazzaro, D. Piparo, G. Schott, W. Verkerke, and M. Wolf, The RooStats project, Proc. Sci., ACAT2010 (2010) 057.

[46] CMS Collaboration, Precise determination of the mass of the Higgs boson and tests of compatibility of its couplings with the standard model predictions using proton collisions at 7 and $8 \mathrm{TeV}$, Eur. Phys. J. C 75, 212 (2015).

[47] G. Cowan, K. Cranmer, E. Gross, and O. Vitells, Asymptotic formulae for likelihood-based tests of new physics, Eur. Phys. J. C 71, 1554 (2011); Erratum, Eur. Phys. J. C 73, 2501 (2011).

[48] M. Czakon and A. Mitov, TOP++: A program for the calculation of the top pair cross section at hadron colliders, Comput. Phys. Commun. 185, 2930 (2014).

[49] M. Czakon, P. Fiedler, and A. Mitov, Total top quark pair production cross section at hadron colliders through $\mathcal{O}\left(\alpha_{\mathrm{S}}^{4}\right)$, Phys. Rev. Lett. 110, 252004 (2013).

[50] R. D. Ball et al. (NNPDF Collaboration), Parton distributions for the LHC Run II, J. High Energy Phys. 04 (2015) 040.

[51] F. Arleo, Tomography of cold and hot QCD matter: Tools and diagnosis, J. High Energy Phys. 11 (2002) 044.

[52] F. Arleo, Quenching of Hadron Spectra in Heavy Ion Collisions at the LHC, Phys. Rev. Lett. 119, 062302 (2017). 
[53] CMS Collaboration, Measurement of Higgs boson production and properties in the WW decay channel with leptonic final states, J. High Energy Phys. 01 (2014) 096.

[54] CMS Collaboration, Jet energy scale and resolution in the CMS experiment in pp collisions at $8 \mathrm{TeV}$, J. Instrum. 12, P02014 (2017).
[55] A. Kusina, F. Lyonnet, D. B. Clark, E. Godat, T. Jezo, K. Kovarik, F. I. Olness, I. Schienbein, and J. Y. Yu, Vector boson production in $p \mathrm{~Pb}$ and $\mathrm{PbPb}$ collisions at the $\mathrm{LHC}$ and its impact on nCTEQ15 PDFs, Eur. Phys. J. C 77, 488 (2017).

A. M. Sirunyan, ${ }^{1, a}$ A. Tumasyan, ${ }^{1}$ W. Adam, ${ }^{2}$ F. Ambrogi,${ }^{2}$ T. Bergauer, ${ }^{2}$ M. Dragicevic, ${ }^{2}$ J. Erö, ${ }^{2}$ A. Escalante Del Valle, ${ }^{2}$ R. Frühwirth, ${ }^{2, b}$ M. Jeitler, ${ }^{2, b}$ N. Krammer, ${ }^{2}$ L. Lechner, ${ }^{2}$ D. Liko, ${ }^{2}$ T. Madlener, ${ }^{2}$ I. Mikulec, ${ }^{2}$ N. Rad, ${ }^{2}$ J. Schieck, ${ }^{2, b}$ R. Schöfbeck, ${ }^{2}$ M. Spanring, ${ }^{2}$ S. Templ, ${ }^{2}$ W. Waltenberger, ${ }^{2}$ C.-E. Wulz, ${ }^{2, b}$ M. Zarucki, ${ }^{2}$ V. Chekhovsky, ${ }^{3}$ A. Litomin, ${ }^{3}$ V. Makarenko, ${ }^{3}$ J. Suarez Gonzalez, ${ }^{3}$ M. R. Darwish, ${ }^{4, c}$ E. A. De Wolf, ${ }^{4}$ D. Di Croce, ${ }^{4}$ X. Janssen, ${ }^{4}$ T. Kello, ${ }^{4, d}$ A. Lelek, ${ }^{4}$ M. Pieters, ${ }^{4}$ H. Rejeb Sfar, ${ }^{4}$ H. Van Haevermaet, ${ }^{4}$ P. Van Mechelen, ${ }^{4}$ S. Van Putte, ${ }^{4}$ N. Van Remortel, ${ }^{4}$ F. Blekman, ${ }^{5}$ E. S. Bols, ${ }^{5}$ S. S. Chhibra, ${ }^{5}$ J. D'Hondt, ${ }^{5}$ J. De Clercq, ${ }^{5}$ D. Lontkovskyi, ${ }^{5}$ S. Lowette, ${ }^{5}$ I. Marchesini, ${ }^{5}$ S. Moortgat, ${ }^{5}$ Q. Python, ${ }^{5}$ S. Tavernier, ${ }^{5}$ W. Van Doninck, ${ }^{5}$ P. Van Mulders, ${ }^{5}$ D. Beghin, ${ }^{6}$ B. Bilin, ${ }^{6}$ B. Clerbaux, ${ }^{6}$ G. De Lentdecker, ${ }^{6}$ H. Delannoy, ${ }^{6}$ B. Dorney, ${ }^{6}$ L. Favart, ${ }^{6}$ A. Grebenyuk, ${ }^{6}$ A. K. Kalsi, ${ }^{6}{ }_{\text {I. Makarenko, }}{ }^{6}$ L. Moureaux, ${ }^{6}$ L. Pétré, ${ }^{6}$ A. Popov, ${ }^{6}$ N. Postiau, ${ }^{6}$ E. Starling, ${ }^{6}$ L. Thomas, ${ }^{6}$ C. Vander Velde, ${ }^{6}$ P. Vanlaer, ${ }^{6}$ D. Vannerom, ${ }^{6}$ L. Wezenbeek, ${ }^{6}$ T. Cornelis, ${ }^{7}$ D. Dobur, ${ }^{7}$ I. Khvastunov, ${ }^{7, e}$ M. Niedziela, ${ }^{7}$ C. Roskas, ${ }^{7}$ K. Skovpen, ${ }^{7}$ M. Tytgat, ${ }^{7}$ W. Verbeke, ${ }^{7}$ B. Vermassen, ${ }^{7}$ M. Vit, ${ }^{7}$ G. Bruno, ${ }^{8}$ F. Bury, ${ }^{8}$ C. Caputo, ${ }^{8}$ P. David,${ }^{8}$ C. Delaere, ${ }^{8}$ M. Delcourt, ${ }^{8}$ I. S. Donertas, ${ }^{8}$ A. Giammanco,${ }^{8}$ V. Lemaitre,${ }^{8}$ J. Prisciandaro, ${ }^{8}$ A. Saggio, ${ }^{8}$ A. Taliercio, ${ }^{8}$ M. Teklishyn,${ }^{8}$ P. Vischia, ${ }^{8}$ S. Wuyckens, ${ }^{8}$ J. Zobec, ${ }^{8}$ G. A. Alves ${ }^{9}$ G. Correia Silva, ${ }^{9}$ C. Hensel, ${ }^{9}$ A. Moraes, ${ }^{9}$ W. L. Aldá Júnior, ${ }^{10}$ E. Belchior Batista Das Chagas, ${ }^{10}$ W. Carvalho, ${ }^{10}$ J. Chinellato, ${ }^{10, \mathrm{f}}$ E. Coelho, ${ }^{10}$ E. M. Da Costa, ${ }^{10}$ G. G. Da Silveira, ${ }^{10, \mathrm{~g}}$ D. De Jesus Damiao, ${ }^{10}$ S. Fonseca De Souza, ${ }^{10}$ H. Malbouisson, ${ }^{10}$ J. Martins, ${ }^{10, h}$ D. Matos Figueiredo, ${ }^{10}$ M. Medina Jaime, ${ }^{10, i}$ M. Melo De Almeida, ${ }^{10}$ C. Mora Herrera, ${ }^{10}$ L. Mundim, ${ }^{10}$ H. Nogima, ${ }^{10}$ P. Rebello Teles, ${ }^{10}$ L. J. Sanchez Rosas, ${ }^{10}$ A. Santoro, ${ }^{10}$ S. M. Silva Do Amaral,${ }^{10}$ A. Sznajder, ${ }^{10}$ M. Thiel,${ }^{10}$ E. J. Tonelli Manganote,${ }^{10, \mathrm{f}}$ F. Torres Da Silva De Araujo, ${ }^{10}$ A. Vilela Pereira, ${ }^{10}$ C. A. Bernardes,${ }^{11 a}$ L. Calligaris, ${ }^{11 \mathrm{a}}$ T. R. Fernandez Perez Tomei, ${ }^{11 \mathrm{a}}$ E. M. Gregores, ${ }^{11 \mathrm{a}, 11 \mathrm{~b}}$ D. S. Lemos, ${ }^{11 \mathrm{a}}$ P. G. Mercadante, ${ }^{11 \mathrm{a}, 11 \mathrm{~b}}$ S. F. Novaes, ${ }^{11 \mathrm{a}}$ Sandra S. Padula, ${ }^{11 \mathrm{a}}$ A. Aleksandrov, ${ }^{12}$ G. Antchev, ${ }^{12}$ I. Atanasov, ${ }^{12}$ R. Hadjiiska, ${ }^{12}$ P. Iaydjiev, ${ }^{12}$ M. Misheva, ${ }^{12}$ M. Rodozov, ${ }^{12}$ M. Shopova, ${ }^{12}$ G. Sultanov, ${ }^{12}$ M. Bonchev, ${ }^{13}$ A. Dimitrov, ${ }^{13}$ T. Ivanov, ${ }^{13}$ L. Litov, ${ }^{13}$ B. Pavlov, ${ }^{13}$ P. Petkov, ${ }^{13}$ A. Petrov, ${ }^{13}$ W. Fang, ${ }^{14, \mathrm{~d}}$ Q. Guo, ${ }^{14}$ H. Wang,${ }^{14}$ L. Yuan, ${ }^{14}$ M. Ahmad, ${ }^{15}$ Z. Hu, ${ }^{15}$ Y. Wang, ${ }^{15}$ E. Chapon, ${ }^{16}$ G. M. Chen, ${ }^{16, j}$ H. S. Chen, ${ }^{16, j}$ M. Chen, ${ }^{16}$ C. H. Jiang, ${ }^{16}$ D. Leggat, ${ }^{16}$ H. Liao, ${ }^{16}$ Z. Liu, ${ }^{16}$ R. Sharma, ${ }^{16}$ A. Spiezia, ${ }^{16}$ J. Tao, ${ }^{16}$ J. Wang, ${ }^{16}$ H. Zhang, ${ }^{16}$ S. Zhang,,${ }^{16, j}$ J. Zhao, ${ }^{16}$ A. Agapitos, ${ }^{17}$ Y. Ban, ${ }^{17}$ C. Chen, ${ }^{17}$ G. Chen, ${ }^{17}$ A. Levin, ${ }^{17}$ J. Li ${ }^{17}$ L. Li,${ }^{17}$ Q. Li,${ }^{17}$ X. Lyu,${ }^{17}$ Y. Mao, ${ }^{17}$ S. J. Qian, ${ }^{17}$ D. Wang,${ }^{17}$ Q. Wang, ${ }^{17}$ J. Xiao, ${ }^{17}$ Z. You, ${ }^{18}$ X. Gao, ${ }^{19, d}$ M. Xiao, ${ }^{20}$ C. Avila, ${ }^{21}$ A. Cabrera, ${ }^{21}$ C. Florez, ${ }^{21}$ J. Fraga, ${ }^{21}$ A. Sarkar, ${ }^{21}$ M. A. Segura Delgado, ${ }^{21}$ J. Mejia Guisao, ${ }^{22}$ F. Ramirez, ${ }^{22}$ J. D. Ruiz Alvarez ${ }^{22}$ C. A. Salazar González, ${ }^{22}$ N. Vanegas Arbelaez, ${ }^{22}$ D. Giljanovic, ${ }^{23}$ N. Godinovic, ${ }^{23}$ D. Lelas, ${ }^{23}$ I. Puljak, ${ }^{23}$ T. Sculac, ${ }^{23}$ Z. Antunovic,${ }^{24}$ M. Kovac, ${ }^{24}$ V. Brigljevic, ${ }^{25}$ D. Ferencek, ${ }^{25}$ D. Majumder,${ }^{25}$ B. Mesic, ${ }^{25}$ M. Roguljic, ${ }^{25}$ A. Starodumov, ${ }^{25, \mathrm{k}}$ T. Susa, ${ }^{25}$ M. W. Ather, ${ }^{26}$ A. Attikis, ${ }^{26}$ E. Erodotou, ${ }^{26}$ A. Ioannou, ${ }^{26}$ G. Kole, ${ }^{26}$ M. Kolosova, ${ }^{26}$ S. Konstantinou, ${ }^{26}$ G. Mavromanolakis, ${ }^{26}$ J. Mousa,${ }^{26}$ C. Nicolaou, ${ }^{26}$ F. Ptochos, ${ }^{26}$ P. A. Razis, ${ }^{26}$ H. Rykaczewski, ${ }^{26}$ H. Saka, ${ }^{26}$ D. Tsiakkouri, ${ }^{26}$ M. Finger, ${ }^{27,1}$ M. Finger Jr., ${ }^{27,1}$ A. Kveton, ${ }^{27}$ J. Tomsa, ${ }^{27}$ E. Ayala, ${ }^{28}$ E. Carrera Jarrin, ${ }^{29}$ E. Salama ${ }^{30, m, n}$ A. Lotfy, ${ }^{31, o}$ M. A. Mahmoud, ${ }^{31}$ S. Bhowmik, ${ }^{32}$ A. Carvalho Antunes De Oliveira, ${ }^{32}$ R. K. Dewanjee, ${ }^{32}$ K. Ehataht, ${ }^{32}$ M. Kadastik, ${ }^{32}$ M. Raidal, ${ }^{32}$ C. Veelken, ${ }^{32}$ P. Eerola, ${ }^{33}$ L. Forthomme, ${ }^{33}$ H. Kirschenmann, ${ }^{33}$ K. Osterberg, ${ }^{33}$ M. Voutilainen, ${ }^{33}$ E. Brücken, ${ }^{34}$ F. Garcia,${ }^{34}$ J. Havukainen, ${ }^{34}$ V. Karimäki,${ }^{34}$ M. S. Kim, ${ }^{34}$ R. Kinnunen, ${ }^{34}$ T. Lampén ${ }^{34}$ K. Lassila-Perini, ${ }^{34}$ S. Laurila, ${ }^{34}$ S. Lehti, ${ }^{34}$ T. Lindén ${ }^{34}$ H. Siikonen, ${ }^{34}$ E. Tuominen, ${ }^{34}$ J. Tuominiemi, ${ }^{34}$ P. Luukka, ${ }^{35}$ T. Tuuva,${ }^{35}$ M. Besancon, ${ }^{36}$ F. Couderc, ${ }^{36}$ M. Dejardin, ${ }^{36}$ D. Denegri, ${ }^{36}$ J. L. Faure, ${ }^{36}$ F. Ferri, ${ }^{36}$ S. Ganjour,${ }^{36}$ A. Givernaud, ${ }^{36}$ P. Gras, ${ }^{36}$ G. Hamel de Monchenault, ${ }^{36}$ P. Jarry,${ }^{36}$ C. Leloup ${ }^{36}$ B. Lenzi, ${ }^{36}$ E. Locci, ${ }^{36}$ J. Malcles, ${ }^{36}$ J. Rander, ${ }^{36}$ A. Rosowsky, ${ }^{36}$ M. Ö. Sahin, ${ }^{36}$ A. Savoy-Navarro, ${ }^{36, p}$ M. Titov,${ }^{36}$ G. B. Yu, ${ }^{36}$ S. Ahuja, ${ }^{37}$ C. Amendola, ${ }^{37}$ F. Beaudette, ${ }^{37}$ M. Bonanomi, ${ }^{37}$ P. Busson, ${ }^{37}$ C. Charlot, ${ }^{37}$ O. Davignon, ${ }^{37}$ B. Diab,${ }^{37}$ G. Falmagne, ${ }^{37}$

R. Granier de Cassagnac, ${ }^{37}$ I. Kucher, ${ }^{37}$ A. Lobanov, ${ }^{37}$ C. Martin Perez, ${ }^{37}$ M. Nguyen, ${ }^{37}$ C. Ochando, ${ }^{37}$ P. Paganini, ${ }^{37}$ J. Rembser,${ }^{37}$ R. Salerno, ${ }^{37}$ J. B. Sauvan, ${ }^{37}$ Y. Sirois,${ }^{37}$ A. Zabi, ${ }^{37}$ A. Zghiche,${ }^{37}$ J.-L. Agram, ${ }^{38, q}$ J. Andrea, ${ }^{38}$ D. Bloch, ${ }^{38}$ G. Bourgatte, ${ }^{38}$ J.-M. Brom, ${ }^{38}$ E. C. Chabert, ${ }^{38}$ C. Collard, ${ }^{38}$ J.-C. Fontaine, ${ }^{38, q}$ D. Gelé, ${ }^{38}$ U. Goerlach, ${ }^{38}$ C. Grimault,${ }^{38}$ 
A.-C. Le Bihan, ${ }^{38}$ P. Van Hove, ${ }^{38}$ E. Asilar, ${ }^{39}$ S. Beauceron, ${ }^{39}$ C. Bernet, ${ }^{39}$ G. Boudoul, ${ }^{39}$ C. Camen, ${ }^{39}$ A. Carle, ${ }^{39}$ N. Chanon, ${ }^{39}$ R. Chierici, ${ }^{39}$ D. Contardo,${ }^{39}$ P. Depasse, ${ }^{39}$ H. El Mamouni, ${ }^{39}$ J. Fay, ${ }^{39}$ S. Gascon, ${ }^{39}$ M. Gouzevitch, ${ }^{39}$ B. Ille, ${ }^{39}$ Sa. Jain, ${ }^{39}$ I. B. Laktineh, ${ }^{39}$ H. Lattaud, ${ }^{39}$ A. Lesauvage, ${ }^{39}$ M. Lethuillier, ${ }^{39}$ L. Mirabito, ${ }^{39}$ L. Torterotot, ${ }^{39}$ G. Touquet, ${ }^{39}$ M. Vander Donckt, ${ }^{39}$ S. Viret, ${ }^{39}$ A. Khvedelidze, ${ }^{40,1}$ Z. Tsamalaidze, ${ }^{41,1}$ L. Feld ${ }^{42}$ K. Klein, ${ }^{42}$ M. Lipinski, ${ }^{42}$ D. Meuser, ${ }^{42}$ A. Pauls,${ }^{42}$ M. Preuten, ${ }^{42}$ M. P. Rauch,${ }^{42}$ J. Schulz ${ }^{42}$ M. Teroerde, ${ }^{42}$ D. Eliseev, ${ }^{43}$ M. Erdmann, ${ }^{43}$ P. Fackeldey, ${ }^{43}$ B. Fischer ${ }^{43}$ S. Ghosh ${ }^{43}$ T. Hebbeker ${ }^{43}$ K. Hoepfner ${ }^{43}$ H. Keller ${ }^{43}$ L. Mastrolorenzo, ${ }^{43}$ M. Merschmeyer, ${ }^{43}$ A. Meyer, ${ }^{43}$ P. Millet, ${ }^{43}$ G. Mocellin, ${ }^{43}$ S. Mondal, ${ }^{43}$ S. Mukherjee, ${ }^{43}$ D. Noll, ${ }^{43}$ A. Novak, ${ }^{43}$ T. Pook, ${ }^{43}$ A. Pozdnyakov,${ }^{43}$ T. Quast ${ }^{43}$ M. Radziej, ${ }^{43}$ Y. Rath, ${ }^{43}$ H. Reithler ${ }^{43}$ J. Roemer, ${ }^{43}$ A. Schmidt, ${ }^{43}$ S. C. Schuler, ${ }^{43}$ A. Sharma, ${ }^{43}$ S. Wiedenbeck, ${ }^{43}$ S. Zaleski, ${ }^{43}$ C. Dziwok, ${ }^{44}$ G. Flügge,${ }^{44}$ W. Haj Ahmad ${ }^{44, r}$ O. Hlushchenko, ${ }^{44}$ T. Kress, ${ }^{44}$ A. Nowack,${ }^{44}$ C. Pistone,${ }^{44}$ O. Pooth, ${ }^{44}$ D. Roy, ${ }^{44}$ H. Sert, ${ }^{44}$ A. Stahl,${ }^{44, s}$ T. Ziemons, ${ }^{44}$ H. Aarup Petersen, ${ }^{45}$ M. Aldaya Martin, ${ }^{45}$ P. Asmuss, ${ }^{45}$ I. Babounikau, ${ }^{45}$ S. Baxter, ${ }^{45}$ O. Behnke, ${ }^{45}$ A. Bermúdez Martínez, ${ }^{45}$ A. A. Bin Anuar, ${ }^{45}$ K. Borras, ${ }^{45, t}$ V. Botta, ${ }^{45}$

D. Brunner, ${ }^{45}$ A. Campbell, ${ }^{45}$ A. Cardini, ${ }^{45}$ P. Connor, ${ }^{45}$ S. Consuegra Rodríguez, ${ }^{45}$ V. Danilov, ${ }^{45}$ A. De Wit, ${ }^{45}$ M. M. Defranchis, ${ }^{45}$ L. Didukh, ${ }^{45}$ D. Domínguez Damiani, ${ }^{45}$ G. Eckerlin, ${ }^{45}$ D. Eckstein, ${ }^{45}$ T. Eichhorn, ${ }^{45}$ A. Elwood, ${ }^{45}$ L. I. Estevez Banos, ${ }^{45}$ E. Gallo, ${ }^{45, u}$ A. Geiser, ${ }^{45}$ A. Giraldi, ${ }^{45}$ A. Grohsjean, ${ }^{45}$ M. Guthoff, ${ }^{45}$ M. Haranko, ${ }^{45}$ A. Harb, ${ }^{45}$ A. Jafari, ${ }^{45, v}$ N. Z. Jomhari, ${ }^{45}$ H. Jung, ${ }^{45}$ A. Kasem, ${ }^{45, t}$ M. Kasemann, ${ }^{45}$ H. Kaveh, ${ }^{45}$ J. Keaveney, ${ }^{45}$ C. Kleinwort, ${ }^{45}$ J. Knolle, ${ }^{45}$ D. Krücker, ${ }^{45}$ W. Lange ${ }^{45}$ T. Lenz,${ }^{45}$ J. Lidrych, ${ }^{45}$ K. Lipka, ${ }^{45}$ W. Lohmann, ${ }^{45, w}$ R. Mankel, ${ }^{45}$

I.-A. Melzer-Pellmann, ${ }^{45}$ J. Metwally, ${ }^{45}$ A. B. Meyer, ${ }^{45}$ M. Meyer, ${ }^{45}$ M. Missiroli, ${ }^{45}$ J. Mnich, ${ }^{45}$ A. Mussgiller, ${ }^{45}$ V. Myronenko, ${ }^{45}$ Y. Otarid, ${ }^{45}$ D. Pérez Adán, ${ }^{45}$ S. K. Pflitsch,${ }^{45}$ D. Pitzl, ${ }^{45}$ A. Raspereza, ${ }^{45}$ A. Saibel,${ }^{45}$ M. Savitskyi, ${ }^{45}$ V. Scheurer, ${ }^{45}$ P. Schütze, ${ }^{45}$ C. Schwanenberger, ${ }^{45}$ R. Shevchenko, ${ }^{45}$ A. Singh, ${ }^{45}$ R. E. Sosa Ricardo, ${ }^{45}$ H. Tholen, ${ }^{45}$ N. Tonon, ${ }^{45}$ O. Turkot, ${ }^{45}$ A. Vagnerini, ${ }^{45}$ M. Van De Klundert, ${ }^{45}$ R. Walsh, ${ }^{45}$ D. Walter, ${ }^{45}$ Y. Wen, ${ }^{45}$ K. Wichmann, ${ }^{45}$ C. Wissing ${ }^{45}$ S. Wuchterl, ${ }^{45}$ O. Zenaiev,${ }^{45}$ R. Zlebcik, ${ }^{45}$ R. Aggleton, ${ }^{46}$ S. Bein, ${ }^{46}$ L. Benato, ${ }^{46}$ A. Benecke, ${ }^{46}$ K. De Leo, ${ }^{46}$ T. Dreyer, ${ }^{46}$ A. Ebrahimi, ${ }^{46}$ F. Feindt, ${ }^{46}$ A. Fröhlich,${ }^{46}$ C. Garbers, ${ }^{46}$ E. Garutti, ${ }^{46}$ D. Gonzalez, ${ }^{46}$ P. Gunnellini, ${ }^{46}$ J. Haller ${ }^{46}$ A. Hinzmann, ${ }^{46}$ A. Karavdina, ${ }^{46}$ G. Kasieczka, ${ }^{46}$ R. Klanner ${ }^{46}$ R. Kogler, ${ }^{46}$ S. Kurz, ${ }^{46}$ V. Kutzner, ${ }^{46}$ J. Lange,${ }^{46}$ T. Lange, ${ }^{46}$ A. Malara, ${ }^{46}$ J. Multhaup, ${ }^{46}$ C. E. N. Niemeyer, ${ }^{46}$ A. Nigamova, ${ }^{46}$ K. J. Pena Rodriguez, ${ }^{46}$ A. Reimers, ${ }^{46}$ O. Rieger, ${ }^{46}$ P. Schleper, ${ }^{46}$ S. Schumann, ${ }^{46}$ J. Schwandt, ${ }^{46}$ D. Schwarz, ${ }^{46}$ J. Sonneveld, ${ }^{46}$ H. Stadie, ${ }^{46}$ G. Steinbrück, ${ }^{46}$ B. Vormwald, ${ }^{46}$

I. Zoi, ${ }^{46}$ M. Akbiyik, ${ }^{47}$ M. Baselga, ${ }^{47}$ S. Baur, ${ }^{47}$ J. Bechtel,${ }^{47}$ T. Berger, ${ }^{47}$ E. Butz, ${ }^{47}$ R. Caspart, ${ }^{47}$ T. Chwalek, ${ }^{47}$ W. De Boer, ${ }^{47}$ A. Dierlamm, ${ }^{47}$ K. El Morabit, ${ }^{47}$ N. Faltermann,${ }^{47}$ K. Flöh, ${ }^{47}$ M. Giffels, ${ }^{47}$ A. Gottmann, ${ }^{47}$ F. Hartmann,,${ }^{47, s}$

C. Heidecker, ${ }^{47}$ U. Husemann, ${ }^{47}$ M. A. Iqbal, ${ }^{47}$ I. Katkov, ${ }^{47, x}$ S. Kudella, ${ }^{47}$ S. Maier, ${ }^{47}$ M. Metzler, ${ }^{47}$ S. Mitra, ${ }^{47}$ M. U. Mozer, ${ }^{47}$ D. Müller, ${ }^{47}$ Th. Müller, ${ }^{47}$ M. Musich,${ }^{47}$ G. Quast, ${ }^{47}$ K. Rabbertz, ${ }^{47}$ J. Rauser, ${ }^{47}$ D. Savoiu, ${ }^{47}$ D. Schäfer, ${ }^{47}$ M. Schnepf, ${ }^{47}$ M. Schröder, ${ }^{47}$ D. Seith, ${ }^{47}$ I. Shvetsov, ${ }^{47}$ H. J. Simonis, ${ }^{47}$ R. Ulrich, ${ }^{47}$ M. Wassmer, ${ }^{47}$ M. Weber, ${ }^{47}$ C. Wöhrmann, ${ }^{47}$ R. Wolf, ${ }^{47}$ S. Wozniewski, ${ }^{47}$ G. Anagnostou, ${ }^{48}$ P. Asenov, ${ }^{48}$ G. Daskalakis, ${ }^{48}$ T. Geralis, ${ }^{48}$ A. Kyriakis, ${ }^{48}$ D. Loukas, ${ }^{48}$ G. Paspalaki, ${ }^{48}$ A. Stakia, ${ }^{48}$ M. Diamantopoulou, ${ }^{49}$ D. Karasavvas, ${ }^{49}$ G. Karathanasis, ${ }^{49}$ P. Kontaxakis,${ }^{49}$

C. K. Koraka, ${ }^{49}$ A. Manousakis-katsikakis, ${ }^{49}$ A. Panagiotou, ${ }^{49}$ I. Papavergou, ${ }^{49}$ N. Saoulidou, ${ }^{49}$ K. Theofilatos, ${ }^{49}$ K. Vellidis, ${ }^{49}$ E. Vourliotis, ${ }^{49}$ G. Bakas,${ }^{50}$ K. Kousouris,${ }^{50}$ I. Papakrivopoulos,${ }^{50}$ G. Tsipolitis, ${ }^{50}$ A. Zacharopoulou, ${ }^{50}$ I. Evangelou, ${ }^{51}$ C. Foudas, ${ }^{51}$ P. Gianneios, ${ }^{51}$ P. Katsoulis, ${ }^{51}$ P. Kokkas, ${ }^{51}$ S. Mallios, ${ }^{51}$ K. Manitara, ${ }^{51}$ N. Manthos, ${ }^{51}$ I. Papadopoulos, ${ }^{51}$ J. Strologas, ${ }^{51}$ D. Tsitsonis, ${ }^{51}$ M. Bartók, ${ }^{52, y}$ R. Chudasama, ${ }^{52}$ M. Csanad ${ }^{52}$ M. M. A. Gadallah, ${ }^{52, z}$ P. Major, ${ }^{52}$ K. Mandal, ${ }^{52}$ A. Mehta, ${ }^{52}$ G. Pasztor, ${ }^{52}$ O. Surányi, ${ }^{52}$ G. I. Veres,${ }^{52}$ G. Bencze, ${ }^{53}$ C. Hajdu, ${ }^{53}$ D. Horvath, ${ }^{53 \text {,aa }}$ F. Sikler, ${ }^{53}$ V. Veszpremi, ${ }^{53}$ G. Vesztergombi, ${ }^{53, a, b b}$ N. Beni, ${ }^{54}$ S. Czellar, ${ }^{54}$ J. Karancsi, ${ }^{54, y}$ J. Molnar, ${ }^{54}$ Z. Szillasi, ${ }^{54}$ D. Teyssier, ${ }^{54}$ P. Raics, ${ }^{55}$ Z. L. Trocsanyi,${ }^{55}$ B. Ujvari, ${ }^{55}$ T. Csorgo, ${ }^{56}$ S. Lököss ${ }^{56, b b}$ F. Nemes,${ }^{56}$ T. Novak, ${ }^{56}$ S. Choudhury, ${ }^{57}$ J. R. Komaragiri, ${ }^{57}$ D. Kumar, ${ }^{57}$ L. Panwar, ${ }^{57}$ P. C. Tiwari, ${ }^{57}$ S. Bahinipati,${ }^{58, c c}$ D. Dash,${ }^{58}$ C. Kar, ${ }^{58}$ P. Mal,${ }^{58}$ T. Mishra,${ }^{58}$ V. K. Muraleedharan Nair Bindhu, ${ }^{58}$ A. Nayak, ${ }^{58, d d}$ D. K. Sahoo, ${ }^{58, c c}$ N. Sur, ${ }^{58}$ S. K. Swain, ${ }^{58}$ S. Bansal, ${ }^{59}$ S. B. Beri, ${ }^{59}$ V. Bhatnagar, ${ }^{59}$ S. Chauhan, ${ }^{59}$ N. Dhingra, ${ }^{59, \text { ee }}$ R. Gupta, ${ }^{59}$ A. Kaur, ${ }^{59}$ A. Kaur, ${ }^{59}$ S. Kaur, ${ }^{59}$ P. Kumari, ${ }^{59}$ M. Lohan, ${ }^{59}$ M. Meena, ${ }^{59}$ K. Sandeep, ${ }^{59}$ S. Sharma, ${ }^{59}$ J. B. Singh, ${ }^{59}$ A. K. Virdi, ${ }^{59}$ A. Ahmed, ${ }^{60}$ A. Bhardwaj, ${ }^{60}$ B. C. Choudhary, ${ }^{60}$ R. B. Garg ${ }^{60}$ M. Gola, ${ }^{60}$ S. Keshri, ${ }^{60}$ A. Kumar, ${ }^{60}$ M. Naimuddin, ${ }^{60}$ P. Priyanka, ${ }^{60}$ K. Ranjan, ${ }^{60}$ A. Shah,${ }^{60}$ M. Bharti, ${ }^{61, f f}$ R. Bhattacharya, ${ }^{61}$ S. Bhattacharya,${ }^{61}$ D. Bhowmik, ${ }^{61}$ S. Dutta,${ }^{61}$ S. Ghosh, ${ }^{61}$ B. Gomber, ${ }^{61, g g}$ M. Maity, ${ }^{61, h h}$ K. Mondal,${ }^{61}$ S. Nandan, ${ }^{61}$ P. Palit, ${ }^{61}$ A. Purohit, ${ }^{61}$ P. K. Rout, ${ }^{61}$ G. Saha ${ }^{61}$ S. Sarkar, ${ }^{61}$ M. Sharan, ${ }^{61}$ B. Singh, ${ }^{61, f f}$ S. Thakur, ${ }^{61, f f}$ P. K. Behera, ${ }^{62}$ S. C. Behera, ${ }^{62}$ P. Kalbhor, ${ }^{62}$ A. Muhammad ${ }^{62}$ R. Pradhan, ${ }^{62}$ P. R. Pujahari, ${ }^{62}$ A. Sharma,${ }^{62}$ A. K. Sikdar, ${ }^{62}$ D. Dutta, ${ }^{63}$ V. Jha, ${ }^{63}$ V. Kumar, ${ }^{63}$ D. K. Mishra, ${ }^{63}$ K. Naskar, ${ }^{63, \text { ii }}$ P. K. Netrakanti, ${ }^{63}$ L. M. Pant, ${ }^{63}$ P. Shukla, ${ }^{63}$ T. Aziz ${ }^{64}$ M. A. Bhat, ${ }^{64}$ S. Dugad, ${ }^{64}$ R. Kumar Verma, ${ }^{64}$ U. Sarkar, ${ }^{64}$ S. Banerjee, ${ }^{65}$ S. Bhattacharya,${ }^{65}$ S. Chatterjee, ${ }^{65}$ P. Das, ${ }^{65}$ 
M. Guchait, ${ }^{65}$ S. Karmakar, ${ }^{65}$ S. Kumar, ${ }^{65}$ G. Majumder, ${ }^{65}$ K. Mazumdar, ${ }^{65}$ S. Mukherjee, ${ }^{65}$ D. Roy, ${ }^{65}$ N. Sahoo, ${ }^{65}$ S. Dube, ${ }^{66}$ B. Kansal, ${ }^{66}$ A. Kapoor, ${ }^{66}$ K. Kothekar, ${ }^{66}$ S. Pandey, ${ }^{66}$ A. Rane ${ }^{66}$ A. Rastogi, ${ }^{66}$ S. Sharma, ${ }^{66}$ H. Bakhshiansohi, ${ }^{67, j j}$ S. Chenarani, ${ }^{68, k k}$ S. M. Etesami, ${ }^{68}$ M. Khakzad, ${ }^{68}$ M. Mohammadi Najafabadi, ${ }^{68}$ M. Naseri, ${ }^{68}$

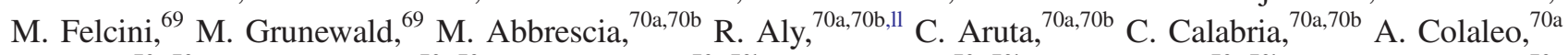
D. Creanza,${ }^{70 a, 70 c}$ N. De Filippis, ${ }^{70 a, 70 c}$ M. De Palma,${ }^{70 a, 70 b}$ A. Di Florio, ${ }^{70 a, 70 b}$ A. Di Pilato, ${ }^{70 a, 70 b}$ W. Elmetenawee, ${ }^{70 a, 70 b}$ L. Fiore, ${ }^{70 a}$ A. Gelmi, ${ }^{70 a, 70 b}$ G. Iaselli, ${ }^{70 a, 70 c}$ M. Ince, ${ }^{70 a, 70 b}$ S. Lezki, ${ }^{70 a, 70 b}$ G. Maggi, ${ }^{70 a, 70 c}$ M. Maggi, ${ }^{70 a}$ I. Margjeka, ${ }^{70 a, 70 b}$ J. A. Merlin, ${ }^{70 \mathrm{a}}$ S. My, ${ }^{70 \mathrm{a}, 70 \mathrm{~b}}$ S. Nuzzo, ${ }^{70 a, 70 b}$ A. Pompili, ${ }^{70 a, 70 b}$ G. Pugliese, ${ }^{70,70 \mathrm{c}}$ A. Ranieri, ${ }^{70 \mathrm{a}}$ G. Selvaggi, ${ }^{70 a, 70 b}$ L. Silvestris, ${ }^{70 a}$ F. M. Simone, ${ }^{70 a, 70 b}$ R. Venditti, ${ }^{70 a}$ P. Verwilligen, ${ }^{70 a}$ G. Abbiendi, ${ }^{71 a}$ C. Battilana, ${ }^{71 a, 71 b}$ D. Bonacorsi, ${ }^{71 a, 71 b}$ L. Borgonovi, ${ }^{71 a, 71 b}$ S. Braibant-Giacomelli, ${ }^{71 a, 71 b}$ R. Campanini, ${ }^{71 a, 71 b}$ P. Capiluppi, ${ }^{71 a, 71 b}$ A. Castro, ${ }^{71 a, 71 b}$ F. R. Cavallo, ${ }^{71 a}$ C. Ciocca, ${ }^{71 a}$ M. Cuffiani, ${ }^{71 a, 71 b}$ G. M. Dallavalle, ${ }^{71 a}$ T. Diotalevi, ${ }^{71 a, 71 b}$ F. Fabbri, ${ }^{71 a}$ A. Fanfani, ${ }^{71 a, 71 b}$ E. Fontanesi, ${ }^{71 a, 71 b}$ P. Giacomelli, ${ }^{71 \mathrm{a}}$ C. Grandi, ${ }^{71 \mathrm{a}}$ L. Guiducci, ${ }^{71 \mathrm{a}, 71 \mathrm{~b}}$ F. Iemmi, ${ }^{71 \mathrm{a}, 71 \mathrm{~b}}$ S. Lo Meo, ${ }^{71 \mathrm{a}, \mathrm{mm}}$ S. Marcellini, ${ }^{71 \mathrm{a}}$ G. Masetti, ${ }^{71 \mathrm{a}}$ F. L. Navarria ${ }^{71 a, 71 b}$ A. Perrotta, ${ }^{71 a}$ F. Primavera, ${ }^{71 a, 71 b}$ A. M. Rossi, ${ }^{71,71 b}$ T. Rovelli, ${ }^{71 a, 71 b}$ G. P. Siroli, ${ }^{71 a, 71 b}$ N. Tosi,${ }^{71 a}$ S. Albergo, ${ }^{72 a, 72 b, n n}$ S. Costa ${ }^{72 a, 72 b}$ A. Di Mattia, ${ }^{72 a}$ R. Potenza, ${ }^{72 a, 72 b}$ A. Tricomi ${ }^{72 a, 72 b, n n}$ C. Tuve, ${ }^{72 a, 72 b}$ G. Barbagli, ${ }^{73 a}$ A. Cassese, ${ }^{73 a}$ R. Ceccarelli, ${ }^{73 a, 73 b}$ V. Ciulli, ${ }^{73 a, 73 b}$ C. Civinini, ${ }^{73 a}$ R. D'Alessandro, ${ }^{73 a, 73 b}$ F. Fiori, ${ }^{73 a}$ E. Focardi, ${ }^{73 a, 73 b}$ G. Latino, ${ }^{73 a, 73 b}$ P. Lenzi, ${ }^{73 a, 73 b}$ M. Lizzo, ${ }^{73 a, 73 b}$ M. Meschini ${ }^{73 a}$ S. Paoletti, ${ }^{73 a}$ R. Seidita, ${ }^{73 a, 73 b}$ G. Sguazzoni, ${ }^{73 a}$ L. Viliani, ${ }^{73 a}$ L. Benussi,${ }^{74}$ S. Bianco,${ }^{74}$ D. Piccolo,${ }^{74}$ M. Bozzo, ${ }^{75 a, 75 b}$ F. Ferro, ${ }^{75 a}$ R. Mulargia, ${ }^{75 a, 75 b}$ E. Robutti, ${ }^{75 a}$ S. Tosi, ${ }^{75 a, 75 b}$ A. Benaglia, ${ }^{76 \mathrm{a}}$ A. Beschi, ${ }^{76 a, 76 b}$ F. Brivio, ${ }^{76 a, 76 b}$ F. Cetorelli, ${ }^{76 a, 76 b}$ V. Ciriolo,${ }^{76 a, 76 b, s}$ F. De Guio, ${ }^{76 a, 76 b}$ M. E. Dinardo, ${ }^{76 a, 76 b}$ P. Dini, ${ }^{76 a}$ S. Gennai, ${ }^{76 a}$ A. Ghezzi,${ }^{76 a, 76 b}$ P. Govoni, ${ }^{76 a, 76 b}$ L. Guzzi, ${ }^{76 a, 76 b}$ M. Malberti, ${ }^{76 a}$ S. Malvezzi ${ }^{76 a}$ D. Menasce, ${ }^{76 a}$ F. Monti, ${ }^{76 a, 76 b}$ L. Moroni, ${ }^{76 a}$ M. Paganoni, ${ }^{76 a, 76 b}$ D. Pedrini, ${ }^{76 a}$ S. Ragazzi, ${ }^{76 a, 76 b}$ T. Tabarelli de Fatis, ${ }^{76 a, 76 b}$

D. Valsecchi, ${ }^{76 a, 76 b, s}$ D. Zuolo, ${ }^{76 a, 76 b}$ S. Buontempo ${ }^{77 a}$ N. Cavallo,${ }^{77 a, 77 c}$ A. De Iorio, ${ }^{77 a, 77 b}$ F. Fabozzi, ${ }^{77 a, 77 c}$ F. Fienga, ${ }^{77 a}$ A. O. M. Iorio, ${ }^{77 a, 77 b}$ L. Layer, ${ }^{77 a, 77 b}$ L. Lista, ${ }^{77 a, 77 b}$ S. Meola, ${ }^{77 a, 77 d, s}$ P. Paolucci, ${ }^{77 a, s}$ B. Rossi, ${ }^{77 a}$ C. Sciacca, ${ }^{77 a, 77 b}$ E. Voevodina, ${ }^{77 a, 77 b}$ P. Azzi, ${ }^{78 a}$ N. Bacchetta, ${ }^{78 a}$ D. Bisello, ${ }^{78 a, 78 b}$ A. Boletti, ${ }^{78 a}, 78 b$ A. Bragagnolo, ${ }^{78 a, 78 b}$ R. Carlin, ${ }^{78 a, 78 b}$ P. Checchia, ${ }^{78 a}$ P. De Castro Manzano, ${ }^{78 a}$ T. Dorigo, ${ }^{78 a}$ U. Dosselli, ${ }^{78 a}$ F. Gasparini, ${ }^{78 a, 78 b}$ U. Gasparini, ${ }^{78 a, 78 b}$ S. Y. Hoh, ${ }^{78 a, 78 b}$ M. Margoni, ${ }^{78 a, 78 b}$ A. T. Meneguzzo, ${ }^{78 a, 78 b}$ M. Presilla, ${ }^{78 a, 78 b}$ P. Ronchese ${ }^{78 a, 78 b}$ R. Rossin ${ }^{78 a, 78 b}$ F. Simonetto, ${ }^{78 a, 78 b}$ G. Strong, ${ }^{78 \mathrm{a}}$ A. Tiko, ${ }^{78 \mathrm{a}}$ M. Tosi, ${ }^{78 \mathrm{a}, 78 \mathrm{~b}}$ M. Zanetti, ${ }^{78 \mathrm{a}, 78 \mathrm{~b}}$ P. Zotto, ${ }^{78 \mathrm{a}, 78 \mathrm{~b}}$ A. Zucchetta, ${ }^{78 \mathrm{a}, 78 \mathrm{~b}}$ G. Zumerle, ${ }^{78 \mathrm{a}, 78 \mathrm{~b}}$ A. Braghieri, ${ }^{79 a}$ S. Calzaferri, ${ }^{79 a, 79 b}$ D. Fiorina, ${ }^{79 a, 79 b}$ P. Montagna,${ }^{79 a, 79 b}$ S. P. Ratti, ${ }^{79 a, 79 b}$ V. Re, ${ }^{79 a}$ M. Ressegotti,${ }^{79 a, 79 b}$ C. Riccardi, ${ }^{79,79 b}$ P. Salvini, ${ }^{79 a}$ I. Vai, ${ }^{79 a}$ P. Vitulo, ${ }^{79 a, 79 b}$ M. Biasini,${ }^{80 a, 80 b}$ G. M. Bilei, ${ }^{80 a}$ D. Ciangottini, ${ }^{80 a, 80 b}$ L. Fanò, ${ }^{80 a, 80 b}$ P. Lariccia ${ }^{80 a, 80 b}$ G. Mantovani, ${ }^{80 a, 80 b}$ V. Mariani, ${ }^{80 a, 80 b}$ M. Menichelli, ${ }^{80 a}$ F. Moscatelli, ${ }^{80 a}$ A. Rossi, ${ }^{80 a, 80 b}$

A. Santocchia ${ }^{80 a, 80 b}$ D. Spiga ${ }^{80 a}$ T. Tedeschi, ${ }^{80 a, 80 b}$ K. Androsov, ${ }^{81 a}$ P. Azzurri, ${ }^{81 a}$ G. Bagliesi, ${ }^{81 a}$ V. Bertacchi, ${ }^{81 a, 81 c}$ L. Bianchini, ${ }^{81 a}$ T. Boccali, ${ }^{81 a}$ R. Castaldi, ${ }^{81 a}$ M. A. Ciocci, ${ }^{81 a, 81 b}$ R. Dell'Orso, ${ }^{81 a}$ M. R. Di Domenico, ${ }^{81 a, 81 b}$ S. Donato, ${ }^{81 a}$ L. Giannini, ${ }^{81 \mathrm{a}, 81 \mathrm{c}}$ A. Giassi, ${ }^{81 \mathrm{a}}$ M. T. Grippo, ${ }^{81 \mathrm{a}}$ F. Ligabue,${ }^{81 \mathrm{a}, 81 \mathrm{c}}$ E. Manca, ${ }^{81 \mathrm{a}, 81 \mathrm{c}}$ G. Mandorli, ${ }^{81 \mathrm{a}, 81 \mathrm{c}}$ A. Messineo, ${ }^{81 \mathrm{a}, 81 \mathrm{~b}}$ F. Palla, ${ }^{81 a}$ A. Rizzi,${ }^{81 a, 81 b}$ G. Rolandi, ${ }^{81 a, 81 c}$ S. Roy Chowdhury, ${ }^{81 a, 81 c}$ A. Scribano, ${ }^{81 a}$ N. Shafiei,${ }^{81 a, 81 b}$ P. Spagnolo, ${ }^{81 a}$ R. Tenchini, ${ }^{81 a}$ G. Tonelli, ${ }^{81 a, 81 b}$ N. Turini, ${ }^{81 a}$ A. Venturi, ${ }^{81 a}$ P. G. Verdini, ${ }^{81 a}$ F. Cavallari, ${ }^{82 a}$ M. Cipriani, ${ }^{82 a, 82 b}$ D. Del Re, ${ }^{82 a, 82 b}$ E. Di Marco, ${ }^{82 a}$ M. Diemoz, ${ }^{82 a}$ E. Longo, ${ }^{82 a, 82 b}$ P. Meridiani, ${ }^{82 a}$ G. Organtini, ${ }^{82 a, 82 b}$ F. Pandolfi, ${ }^{82 a}$ R. Paramatti, ${ }^{82 a, 82 b}$ C. Quaranta, ${ }^{82 a, 82 b}$ S. Rahatlou, ${ }^{82 a, 82 b}$ C. Rovelli, ${ }^{82 a}$ F. Santanastasio, ${ }^{82 a, 82 b}$ L. Soffi, ${ }^{82 a, 82 b}$

R. Tramontano, ${ }^{82 a, 82 b}$ N. Amapane ${ }^{83 a, 83 b}$ R. Arcidiacono, ${ }^{83 a, 83 c}$ S. Argiro, ${ }^{83 a, 83 b}$ M. Arneodo ${ }^{83 a, 83 c}$ N. Bartosik ${ }^{83 a}$ R. Bellan, ${ }^{83 a, 83 b}$ A. Bellora, ${ }^{83 a, 83 b}$ C. Biino ${ }^{83 a}$ A. Cappati, ${ }^{83 a, 83 b}$ N. Cartigliaa, ${ }^{83 a}$ S. Cometti, ${ }^{83 a}$ M. Costa, ${ }^{83 a, 83 b}$ R. Covarelli, ${ }^{83 a, 83 b}$ N. Demaria, ${ }^{83 a}$ B. Kiani, ${ }^{83 a, 83 b}$ F. Legger, ${ }^{83 a}$ C. Mariotti, ${ }^{83 a}$ S. Maselli, ${ }^{83 a}$ E. Migliore, ${ }^{83 a, 83 b}$ V. Monaco, ${ }^{83 a, 83 b}$ E. Monteil, ${ }^{83 a, 83 b}$ M. Monteno, ${ }^{83 a}$ M. M. Obertino, ${ }^{83 a, 83 b}$ G. Ortona, ${ }^{83 a}$ L. Pacher, ${ }^{83 a, 83 b}$ N. Pastrone ${ }^{83 a}$ M. Pelliccioni, ${ }^{83 a}$ G. L. Pinna Angioni, ${ }^{83 a, 83 b}$ M. Ruspa,${ }^{83 a, 83 c}$ R. Salvatico, ${ }^{83 a, 83 b}$ F. Siviero, ${ }^{83 a, 83 b}$ V. Sola, ${ }^{83 a}$ A. Solano, ${ }^{83 a, 83 b}$ D. Soldi ${ }^{83 a, 83 b}$ A. Staiano, ${ }^{83 a}$ D. Trocino, ${ }^{83 a, 83 b}$ S. Belforte, ${ }^{84 a}$ V. Candelise,${ }^{84 a, 84 b}$ M. Casarsa, ${ }^{84 a}$ F. Cossutti,${ }^{84 a}$ A. Da Rold ${ }^{84 a, 84 b}$ G. Della Ricca, ${ }^{84 a, 84 b}$ F. Vazzoler, ${ }^{84 a, 84 b}$ S. Dogra, ${ }^{85}$ C. Huh, ${ }^{85}$ B. Kim, ${ }^{85}$ D. H. Kim, ${ }^{85}$ G. N. Kim,${ }^{85}$ J. Lee, ${ }^{85}$ S. W. Lee, ${ }^{85}$ C. S. Moon, ${ }^{85}$ Y. D. Oh ${ }^{85}$ S. I. Pak ${ }^{85}$ S. Sekmen,${ }^{85}$ Y. C. Yang, ${ }^{85}$ H. Kim, ${ }^{86}$ D. H. Moon, ${ }^{86}$ B. Francois, ${ }^{87}$ T. J. Kim, ${ }^{87}$ J. Park, ${ }^{87}$ S. Cho, ${ }^{88}$ S. Choi,${ }^{88}$ Y. Go, ${ }^{88}$ S. Ha ${ }^{88}$ B. Hong ${ }^{88}$ K. Lee, ${ }^{88}$ K. S. Lee,${ }^{88}$ J. Lim, ${ }^{88}$ J. Park, ${ }^{88}$ S. K. Park, ${ }^{88}$ Y. Roh, ${ }^{88}$ J. Yoo ${ }^{88}$ J. Goh, ${ }^{89}$ A. Gurtu, ${ }^{89}$ H. S. Kim, ${ }^{90}$ Y. Kim, ${ }^{90}$ J. Almond, ${ }^{91}$ J. H. Bhyun, ${ }^{91}$ J. Choi, ${ }^{91}$ S. Jeon,,${ }^{91}$ J. Kim, ${ }^{11}$ J. S. Kim, ${ }^{91}$ S. Ko, ${ }^{11}$ H. Kwon, ${ }^{91}$ H. Lee, ${ }^{91}$ K. Lee, ${ }^{91}$ S. Lee, ${ }^{91}$ K. Nam, ${ }^{91}$ B. H. Oh, ${ }^{11}$ M. Oh, ${ }^{91}$ S. B. Oh,${ }^{91}$ B. C. Radburn-Smith, ${ }^{91}$ H. Seo, ${ }^{91}$ U. K. Yang, ${ }^{91}$ I. Yoon, ${ }^{91}$ D. Jeon, ${ }^{92}$ J. H. Kim, ${ }^{92}$ B. Ko, ${ }^{92}$ J. S. H. Lee, ${ }^{92}$ I. C. Park, ${ }^{92}$ I. J. Watson, ${ }^{92}$ H. D. Yoo, ${ }^{93}$ Y. Choi,${ }^{94}$ C. Hwang, ${ }^{94}$ Y. Jeong, ${ }^{94}$ H. Lee,${ }^{94}$ J. Lee, ${ }^{94}$ Y. Lee ${ }^{94}$ I. Yu, ${ }^{94}$ V. Veckalns, ${ }^{95,00}$ A. Juodagalvis, ${ }^{96}$ A. Rinkevicius, ${ }^{96}$ G. Tamulaitis, ${ }^{96}$ W. A. T. Wan Abdullah, ${ }^{97}$ M. N. Yusli, ${ }^{97}$ Z. Zolkapli, ${ }^{97}$ J. F. Benitez, ${ }^{98}$ 
A. Castaneda Hernandez, ${ }^{98}$ J. A. Murillo Quijada, ${ }^{98}$ L. Valencia Palomo, ${ }^{98}$ H. Castilla-Valdez, ${ }^{99}$ E. De La Cruz-Burelo, ${ }^{99}$ I. Heredia-De La Cruz,${ }^{99, p p}$ R. Lopez-Fernandez ${ }^{99}$ A. Sanchez-Hernandez, ${ }^{99}$ S. Carrillo Moreno, ${ }^{100}$ C. Oropeza Barrera, ${ }^{100}$ M. Ramirez-Garcia, ${ }^{100}$ F. Vazquez Valencia, ${ }^{100}$ J. Eysermans, ${ }^{101}$ I. Pedraza, ${ }^{101}$ H. A. Salazar Ibarguen, ${ }^{101}$ C. Uribe Estrada, ${ }^{101}$ A. Morelos Pineda, ${ }^{102}$ J. Mijuskovic, ${ }^{103, e}$ N. Raicevic, ${ }^{103}$ D. Krofcheck, ${ }^{104}$ S. Bheesette, ${ }^{105}$ P. H. Butler, ${ }^{105}$ A. Ahmad, ${ }^{106}$ M. I. Asghar, ${ }^{106}$ M. I. M. Awan, ${ }^{106}$ Q. Hassan,${ }^{106}$ H. R. Hoorani, ${ }^{106}$ W. A. Khan, ${ }^{106}$ M. A. Shah, ${ }^{106}$ M. Shoaib, ${ }^{106}$ M. Waqas, ${ }^{106}$ V. Avati, ${ }^{107}$ L. Grzanka, ${ }^{107}$ M. Malawski, ${ }^{107}$ H. Bialkowska, ${ }^{108}$ M. Bluj, ${ }^{108}$ B. Boimska, ${ }^{108}$ T. Frueboes, ${ }^{108}$ M. Górski, ${ }^{108}$ M. Kazana, ${ }^{108}$ M. Szleper, ${ }^{108}$ P. Traczyk, ${ }^{108}$ P. Zalewski,${ }^{108}$ K. Bunkowski, ${ }^{109}$ A. Byszuk, ${ }^{109, q q}$ K. Doroba, ${ }^{109}$ A. Kalinowski, ${ }^{109}$ M. Konecki, ${ }^{109}$ J. Krolikowski, ${ }^{109}$ M. Olszewski, ${ }^{109}$ M. Walczak, ${ }^{109}$ M. Araujo, ${ }^{110}$ P. Bargassa, ${ }^{110}$ D. Bastos, ${ }^{110}$ A. Di Francesco, ${ }^{110}$ P. Faccioli, ${ }^{110}$ B. Galinhas, ${ }^{110}$ M. Gallinaro, ${ }^{110}$ J. Hollar, ${ }^{110}$ N. Leonardo, ${ }^{110}$ T. Niknejad, ${ }^{110}$ J. Seixas, ${ }^{110}$ K. Shchelina, ${ }^{110}$ O. Toldaiev, ${ }^{110}$ J. Varela, ${ }^{110}$ S. Afanasiev,${ }^{111}$ M. Gavrilenko, ${ }^{111}$

A. Golunov, ${ }^{111}$ I. Golutvin, ${ }^{111}$ N. Gorbounov, ${ }^{111}$ I. Gorbunov, ${ }^{111}$ A. Kamenev, ${ }^{111}$ V. Karjavine, ${ }^{111}$ V. Korenkov, ${ }^{111}$ A. Lanev, ${ }^{111}$ A. Malakhov, ${ }^{111}$ V. Matveev,${ }^{11, \text { rr,ss }}$ P. Moisenz,${ }^{111}$ V. Palichik, ${ }^{111}$ V. Perelygin, ${ }^{111}$ D. Seitova, ${ }^{111}$ S. Shmatov, ${ }^{111}$ V. Smirnov, ${ }^{111}$ O. Teryaev, ${ }^{111}$ N. Voytishin, ${ }^{111}$ A. Zarubin, ${ }^{111}$ G. Gavrilov, ${ }^{112}$ V. Golovtcov, ${ }^{112}$ Y. Ivanov, ${ }^{112}$ V. Kim, ${ }^{112, t t}$ E. Kuznetsova, ${ }^{12, \text { uu }}$ V. Murzin, ${ }^{112}$ V. Oreshkin, ${ }^{12}$ I. Smirnov, ${ }^{112}$ D. Sosnov, ${ }^{112}$ V. Sulimov, ${ }^{112}$ L. Uvarov, ${ }^{112}$ S. Volkov, ${ }^{112}$ A. Vorobyev, ${ }^{112}$ Yu. Andreev, ${ }^{113}$ A. Dermenev, ${ }^{113}$ S. Gninenko, ${ }^{113}$ N. Golubev, ${ }^{113}$ A. Karneyeu,${ }^{113}$ M. Kirsanov,${ }^{113}$

N. Krasnikov, ${ }^{113}$ A. Pashenkov, ${ }^{113}$ G. Pivovarov, ${ }^{113}$ D. Tlisov, ${ }^{113}$ A. Toropin, ${ }^{113}$ V. Epshteyn, ${ }^{114}$ V. Gavrilov, ${ }^{114}$ N. Lychkovskaya, ${ }^{114}$ A. Nikitenko, ${ }^{114, v v}$ V. Popov, ${ }^{114}$ I. Pozdnyakov, ${ }^{114}$ G. Safronov, ${ }^{114}$ A. Spiridonov, ${ }^{114}$ A. Stepennov, ${ }^{114}$ M. Toms, ${ }^{114}$ E. Vlasov, ${ }^{114}$ A. Zhokin, ${ }^{114}$ T. Aushev, ${ }^{115}$ R. Chistov, ${ }^{116, w w}$ M. Danilov, ${ }^{116, w w}$ P. Parygin, ${ }^{116}$ D. Philippov, ${ }^{116}$

S. Polikarpov, ${ }^{16, \text { ww }}$ V. Andreev, ${ }^{117}$ M. Azarkin, ${ }^{117}$ I. Dremin,${ }^{117}$ M. Kirakosyan, ${ }^{117}$ A. Terkulov, ${ }^{117}$ A. Baskakov, ${ }^{118}$ A. Belyaev, ${ }^{118}$ E. Boos, ${ }^{118}$ L. Dudko, ${ }^{118}$ A. Ershov, ${ }^{118}$ A. Gribushin, ${ }^{118}$ O. Kodolova, ${ }^{118}$ V. Korotkikh, ${ }^{118}$ I. Lokhtin, ${ }^{118}$ S. Obraztsov, ${ }^{118}$ S. Petrushanko, ${ }^{118}$ V. Savrin, ${ }^{118}$ A. Snigirev, ${ }^{118}$ V. Blinov, ${ }^{119, x x}$ T. Dimova, ${ }^{119, x x}$ L. Kardapoltsev, ${ }^{11, x x}$ I. Ovtin, ${ }^{11, x x}$ Y. Skovpen, ${ }^{119, x x}$ I. Azhgirey, ${ }^{120}$ I. Bayshev, ${ }^{120}$ V. Kachanov, ${ }^{120}$ A. Kalinin, ${ }^{120}$ D. Konstantinov, ${ }^{120}$ V. Petrov, ${ }^{120}$ R. Ryutin, ${ }^{120}$ A. Sobol, ${ }^{120}$ S. Troshin, ${ }^{120}$ N. Tyurin, ${ }^{120}$ A. Uzunian, ${ }^{120}$ A. Volkov, ${ }^{120}$ A. Babaev, ${ }^{121}$ A. Iuzhakov, ${ }^{121}$ V. Okhotnikov, ${ }^{121}$ V. Borchsh, ${ }^{122}$ V. Ivanchenko, ${ }^{122}$ E. Tcherniaev, ${ }^{122}$ P. Adzic, ${ }^{123, y y}$ P. Cirkovic, ${ }^{123}$ M. Dordevic, ${ }^{123}$ P. Milenovic, ${ }^{123}$ J. Milosevic, ${ }^{123}$ M. Stojanovic, ${ }^{123}$ M. Aguilar-Benitez, ${ }^{124}$ J. Alcaraz Maestre, ${ }^{124}$ A. Álvarez Fernández, ${ }^{124}$ I. Bachiller, ${ }^{124}$ M. Barrio Luna, ${ }^{124}$ J. A. Brochero Cifuentes, ${ }^{124}$ C. A. Carrillo Montoya, ${ }^{124}$ M. Cepeda, ${ }^{124}$ M. Cerrada, ${ }^{124}$ N. Colino, ${ }^{124}$ B. De La Cruz, ${ }^{124}$ A. Delgado Peris, ${ }^{124}$ C. Fernandez Bedoya, ${ }^{124}$ J. P. Fernández Ramos, ${ }^{124}$ J. Flix ${ }^{124}$ M. C. Fouz, ${ }^{124}$ O. Gonzalez Lopez, ${ }^{124}$ S. Goy Lopez, ${ }^{124}$ J. M. Hernandez, ${ }^{124}$ M. I. Josa, ${ }^{124}$ D. Moran, ${ }^{124}$ Á. Navarro Tobar, ${ }^{124}$ A. Pérez-Calero Yzquierdo, ${ }^{124}$ J. Puerta Pelayo, ${ }^{124}$ I. Redondo, ${ }^{124}$ L. Romero, ${ }^{124}$ S. Sánchez Navas, ${ }^{124}$ M. S. Soares, ${ }^{124}$ A. Triossi, ${ }^{124}$ C. Willmott, ${ }^{124}$ C. Albajar, ${ }^{125}$ J. F. de Trocóniz, ${ }^{125}$ R. Reyes-Almanza, ${ }^{125}$ B. Alvarez Gonzalez, ${ }^{126}$ J. Cuevas, ${ }^{126}$ C. Erice, ${ }^{126}$ J. Fernandez Menendez, ${ }^{126}$ S. Folgueras, ${ }^{126}$ I. Gonzalez Caballero, ${ }^{126}$ E. Palencia Cortezon, ${ }^{126}$ C. Ramón Álvarez, ${ }^{126}$ V. Rodríguez Bouza, ${ }^{126}$ S. Sanchez Cruz, ${ }^{126}$ I. J. Cabrillo, ${ }^{127}$ A. Calderon, ${ }^{127}$ B. Chazin Quero, ${ }^{127}$ J. Duarte Campderros, ${ }^{127}$ M. Fernandez, ${ }^{127}$ P. J. Fernández Manteca, ${ }^{127}$ A. García Alonso, ${ }^{127}$ G. Gomez, ${ }^{127}$ C. Martinez Rivero, ${ }^{127}$ P. Martinez Ruiz del Arbol,${ }^{127}$ F. Matorras, ${ }^{127}$ J. Piedra Gomez, ${ }^{127}$ C. Prieels, ${ }^{127}$ F. Ricci-Tam, ${ }^{127}$ T. Rodrigo, ${ }^{127}$ A. Ruiz-Jimeno, ${ }^{127}$ L. Russo, ${ }^{127, z z}$ L. Scodellaro, ${ }^{127}$ I. Vila, ${ }^{127}$ J. M. Vizan Garcia, ${ }^{127}$ MK Jayananda, ${ }^{128}$ B. Kailasapathy, ${ }^{128, \text { aaa }}$ D. U. J. Sonnadara, ${ }^{128}$ DDC Wickramarathna, ${ }^{128}$ W. G. D. Dharmaratna, ${ }^{129}$ K. Liyanage, ${ }^{129}$ N. Perera, ${ }^{129}$ N. Wickramage, ${ }^{129}$ T. K. Aarrestad ${ }^{130}$ D. Abbaneo,${ }^{130}$ B. Akgun, ${ }^{130}$ E. Auffray, ${ }^{130}$ G. Auzinger, ${ }^{130}$ J. Baechler, ${ }^{130}$ P. Baillon, ${ }^{130}$ A. H. Ball, ${ }^{130}$ D. Barney, ${ }^{130}$ J. Bendavid, ${ }^{130}$ M. Bianco, ${ }^{130}$ A. Bocci, ${ }^{130}$ P. Bortignon, ${ }^{130}$ E. Bossini, ${ }^{130}$ E. Brondolin, ${ }^{130}$ T. Camporesi,${ }^{130}$ G. Cerminara, ${ }^{130}$ L. Cristella, ${ }^{130}$ D. d'Enterria, ${ }^{130}$ A. Dabrowski, ${ }^{130}$ N. Daci ${ }^{130}$ V. Daponte, ${ }^{130}$ A. David ${ }^{130}$ A. De Roeck, ${ }^{130}$ M. Deile, ${ }^{130}$ R. Di Maria, ${ }^{130}$ M. Dobson, ${ }^{130}$ M. Dünser, ${ }^{130}$ N. Dupont, ${ }^{130}$ A. Elliott-Peisert, ${ }^{130}$ N. Emriskova, ${ }^{130}$ F. Fallavollita, ${ }^{130, b b b}$ D. Fasanella, ${ }^{130}$ S. Fiorendi, ${ }^{130}$ G. Franzoni, ${ }^{130}$ J. Fulcher, ${ }^{130}$ W. Funk, ${ }^{130}$ S. Giani, ${ }^{130}$ D. Gigi,${ }^{130}$ K. Gill, ${ }^{130}$ F. Glege, ${ }^{130}$ L. Gouskos, ${ }^{130}$ M. Gruchala ${ }^{130}$ M. Guilbaud, ${ }^{130}$ D. Gulhan, ${ }^{130}$ J. Hegeman, ${ }^{130}$ Y. liyama,${ }^{130}$ V. Innocente, ${ }^{130}$ T. James,${ }^{130}$ P. Janot, ${ }^{130}$ J. Kaspar, ${ }^{130}$ J. Kieseler, ${ }^{130}$ M. Komm, ${ }^{130}$ N. Kratochwil,${ }^{130}$ C. Lange,${ }^{130}$ P. Lecoq ${ }^{130}$ K. Long, ${ }^{130}$ C. Lourenço, ${ }^{130}$ L. Malgeri, ${ }^{130}$ M. Mannelli, ${ }^{130}$ A. Massironi, ${ }^{130}$ F. Meijers, ${ }^{130}$ S. Mersi, ${ }^{130}$ E. Meschi, ${ }^{130}$ F. Moortgat ${ }^{130}$ M. Mulders, ${ }^{130}$ J. Ngadiuba ${ }^{130}$ J. Niedziela, ${ }^{130}$ S. Orfanelli, ${ }^{130}$ L. Orsini,${ }^{130}$ F. Pantaleo, ${ }^{130, \mathrm{~s}}$ L. Pape,${ }^{130}$ E. Perez, ${ }^{130}$ M. Peruzzi, ${ }^{130}$ A. Petrilli, ${ }^{130}$ G. Petrucciani, ${ }^{130}$ A. Pfeiffer, ${ }^{130}$ M. Pierini, ${ }^{130}$ F. M. Pitters,${ }^{130}$ D. Rabady, ${ }^{130}$ A. Racz, ${ }^{130}$ M. Rieger, ${ }^{130}$ M. Rovere, ${ }^{130}$ H. Sakulin, ${ }^{130}$ J. Salfeld-Nebgen, ${ }^{130}$ S. Scarfi,${ }^{130}$ C. Schäfer,${ }^{130}$ C. Schwick, ${ }^{130}$ M. Selvaggi,${ }^{130}$ A. Sharma, ${ }^{130}$ P. Silva ${ }^{130}$ W. Snoeys, ${ }^{130}$ P. Sphicas, ${ }^{130, c c c}$ J. Steggemann, ${ }^{130}$ S. Summers, ${ }^{130}$ V. R. Tavolaro, ${ }^{130}$ D. Treille,${ }^{130}$ A. Tsirou, ${ }^{130}$ 
G. P. Van Onsem, ${ }^{130}$ A. Vartak, ${ }^{130}$ M. Verzetti, ${ }^{130}$ K. A. Wozniak, ${ }^{130}$ W. D. Zeuner, ${ }^{130}$ L. Caminada, ${ }^{131, d d d}$ W. Erdmann, ${ }^{131}$ R. Horisberger, ${ }^{131}$ Q. Ingram, ${ }^{131}$ H. C. Kaestli, ${ }^{131}$ D. Kotlinski, ${ }^{131}$ U. Langenegger, ${ }^{131}$ T. Rohe, ${ }^{131}$ M. Backhaus, ${ }^{132}$ P. Berger, ${ }^{132}$ A. Calandri, ${ }^{132}$ N. Chernyavskaya, ${ }^{132}$ G. Dissertori, ${ }^{132}$ M. Dittmar, ${ }^{132}$ M. Donegà, ${ }^{132}$ C. Dorfer, ${ }^{132}$ T. Gadek, ${ }^{132}$ T. A. Gómez Espinosa, ${ }^{132}$ C. Grab, ${ }^{132}$ D. Hits, ${ }^{132}$ W. Lustermann, ${ }^{132}$ A.-M. Lyon, ${ }^{132}$ R. A. Manzoni, ${ }^{132}$ M. T. Meinhard, ${ }^{132}$ F. Micheli, ${ }^{132}$ P. Musella, ${ }^{132}$ F. Nessi-Tedaldi, ${ }^{132}$ F. Pauss, ${ }^{132}$ V. Perovic, ${ }^{132}$ G. Perrin, ${ }^{132}$ L. Perrozzi, ${ }^{132}$ S. Pigazzini ${ }^{132}$ M. G. Ratti, ${ }^{132}$ M. Reichmann, ${ }^{132}$ C. Reissel, ${ }^{132}$ T. Reitenspiess, ${ }^{132}$ B. Ristic, ${ }^{132}$ D. Ruini, ${ }^{132}$ D. A. Sanz Becerra, ${ }^{132}$ M. Schönenberger, ${ }^{132}$ L. Shchutska, ${ }^{132}$ V. Stampf,${ }^{132}$ M. L. Vesterbacka Olsson, ${ }^{132}$ R. Wallny, ${ }^{132}$ D. H. Zhu, ${ }^{132}$ C. Amsler, ${ }^{133, \text { eee }}$ C. Botta, ${ }^{133}$ D. Brzhechko, ${ }^{133}$ M. F. Canelli, ${ }^{133}$ A. De Cosa,${ }^{133}$ R. Del Burgo,${ }^{133}$ J. K. Heikkilä, ${ }^{133}$ M. Huwiler, ${ }^{133}$ A. Jofrehei, ${ }^{133}$ B. Kilminster, ${ }^{133}$ S. Leontsinis, ${ }^{133}$ A. Macchiolo, ${ }^{133}$ V. M. Mikuni, ${ }^{133}$ U. Molinatti, ${ }^{133}$ I. Neutelings, ${ }^{133}$ G. Rauco, ${ }^{133}$ P. Robmann, ${ }^{133}$ K. Schweiger, ${ }^{133}$ Y. Takahashi, ${ }^{133}$ S. Wertz, ${ }^{133}$ C. Adloff, ${ }^{134, f f f}$ C. M. Kuo, ${ }^{134}$ W. Lin, ${ }^{134}$ A. Roy, ${ }^{134}$ T. Sarkar, ${ }^{134, h h}$ S. S. Yu, ${ }^{134}$ L. Ceard,${ }^{135}$ P. Chang, ${ }^{135}$ Y. Chao, ${ }^{135}$ K. F. Chen, ${ }^{135}$ P. H. Chen, ${ }^{135}$ W.-S. Hou, ${ }^{135}$ Y. y. Li, ${ }^{135}$ R.-S. Lu, ${ }^{135}$ E. Paganis, ${ }^{135}$ A. Psallidas, ${ }^{135}$ A. Steen, ${ }^{135}$ E. Yazgan, ${ }^{135}$ B. Asavapibhop, ${ }^{136}$ C. Asawatangtrakuldee, ${ }^{136}$ N. Srimanobhas, ${ }^{136}$ F. Boran, ${ }^{137}$ S. Damarseckin, ${ }^{137, g g g}$ Z. S. Demiroglu, ${ }^{137}$ F. Dolek, ${ }^{137}$ C. Dozen, ${ }^{137, \text { hhh }}$ I. Dumanoglu, ${ }^{137, \text { iii }}$ E. Eskut, ${ }^{137}$ G. Gokbulut, ${ }^{137}$ Y. Guler, ${ }^{137}$ E. Gurpinar Guler, ${ }^{137, j j j}$ I. Hos, ${ }^{137, k k k}$ C. Isik, ${ }^{137}$ E. E. Kangal, ${ }^{137,111}$ O. Kara, ${ }^{137}$ A. Kayis Topaksu, ${ }^{137}$ U. Kiminsu, ${ }^{137}$ G. Onengut, ${ }^{137}$ K. Ozdemir, ${ }^{137, m m m}$ A. Polatoz, ${ }^{137}$ A. E. Simsek, ${ }^{137}$ B. Tali, ${ }^{137, n n n}$ U. G. Tok,${ }^{137}$ S. Turkcapar, ${ }^{137}$ I. S. Zorbakir, ${ }^{137}$ C. Zorbilmez, ${ }^{137}$ B. Isildak, ${ }^{138,000}$ G. Karapinar, ${ }^{138, p p p}$ K. Ocalan, ${ }^{138, q q q}$ M. Yalvac, ${ }^{138, \text { rrr }}$ I. O. Atakisi,${ }^{139}$ E. Gülmez, ${ }^{139}$ M. Kaya ${ }^{139, \text { sss }}$ O. Kaya ${ }^{139, \text { ttt }}$ Ö. Özçelik, ${ }^{139}$ S. Tekten, ${ }^{139, \text { uuu }}$ E. A. Yetkin, ${ }^{139, \text { vvv }}$ A. Cakir, ${ }^{140}$ K. Cankocak, ${ }^{140, i i i}$ Y. Komurcu, ${ }^{140}$ S. Sen, ${ }^{140, \text { www }}$ F. Aydogmus Sen, ${ }^{141}$ S. Cerci, ${ }^{141, n n n}$ B. Kaynak, ${ }^{141}$ S. Ozkorucuklu, ${ }^{141}$ D. Sunar Cerci,${ }^{141, n n n}$ B. Grynyov, ${ }^{142}$ L. Levchuk, ${ }^{143}$ E. Bhal, ${ }^{144}$ S. Bologna, ${ }^{144}$ J. J. Brooke, ${ }^{144}$ D. Burns,${ }^{144, x x x}$ E. Clement, ${ }^{144}$ D. Cussans, ${ }^{144}$ H. Flacher, ${ }^{144}$ J. Goldstein, ${ }^{144}$ G. P. Heath, ${ }^{144}$ H. F. Heath, ${ }^{144}$ L. Kreczko, ${ }^{144}$ B. Krikler, ${ }^{144}$ S. Paramesvaran, ${ }^{144}$ T. Sakuma, ${ }^{144}$ S. Seif El Nasr-Storey, ${ }^{144}$ V. J. Smith, ${ }^{144}$ J. Taylor, ${ }^{144}$ A. Titterton, ${ }^{144}$ K. W. Bell, ${ }^{145}$ A. Belyaev,${ }^{145, y y y}$ C. Brew, ${ }^{145}$ R. M. Brown, ${ }^{145}$ D. J. A. Cockerill, ${ }^{145}$ K. V. Ellis, ${ }^{145}$ K. Harder, ${ }^{145}$ S. Harper, ${ }^{145}$ J. Linacre, ${ }^{145}$ K. Manolopoulos,${ }^{145}$ D. M. Newbold, ${ }^{145}$

E. Olaiya, ${ }^{145}$ D. Petyt, ${ }^{145}$ T. Reis, ${ }^{145}$ T. Schuh, ${ }^{145}$ C. H. Shepherd-Themistocleous, ${ }^{145}$ A. Thea, ${ }^{145}$ I. R. Tomalin, ${ }^{145}$ T. Williams, ${ }^{145}$ R. Bainbridge ${ }^{146}$ P. Bloch,${ }^{146}$ S. Bonomally, ${ }^{146}$ J. Borg, ${ }^{146}$ S. Breeze, ${ }^{146}$ O. Buchmuller,${ }^{146}$ A. Bundock, ${ }^{146}$ V. Cepaitis, ${ }^{146}$ G. S. Chahal, ${ }^{146, z z z}$ D. Colling, ${ }^{146}$ P. Dauncey, ${ }^{146}$ G. Davies, ${ }^{146}$ M. Della Negra,${ }^{146}$ P. Everaerts, ${ }^{146}$ G. Fedi, ${ }^{146}$ G. Hall, ${ }^{146}$ G. Iles, ${ }^{146}$ J. Langford, ${ }^{146}$ L. Lyons, ${ }^{146}$ A.-M. Magnan, ${ }^{146}$ S. Malik, ${ }^{146}$ A. Martelli, ${ }^{146}$ V. Milosevic, ${ }^{146}$ A. Morton, ${ }^{146}$ J. Nash, ${ }^{146, \text { aaaa }}$ V. Palladino, ${ }^{146}$ M. Pesaresi, ${ }^{146}$ D. M. Raymond, ${ }^{146}$ A. Richards, ${ }^{146}$ A. Rose, ${ }^{146}$ E. Scott ${ }^{146}$ C. Seez, ${ }^{146}$ A. Shtipliyski, ${ }^{146}$ M. Stoye,${ }^{146}$ A. Tapper, ${ }^{146}$ K. Uchida, ${ }^{146}$ T. Virdee,${ }^{146, s}$ N. Wardle, ${ }^{146}$ S. N. Webb, ${ }^{146}$ D. Winterbottom, ${ }^{146}$ A. G. Zecchinelli, ${ }^{146}$ S. C. Zenz, ${ }^{146}$ J. E. Cole,${ }^{147}$ P. R. Hobson, ${ }^{147}$ A. Khan, ${ }^{147}$ P. Kyberd, ${ }^{147}$ C. K. Mackay, ${ }^{147}$ I. D. Reid, ${ }^{147}$ L. Teodorescu, ${ }^{147}$ S. Zahid, ${ }^{147}$ A. Brinkerhoff, ${ }^{148}$ K. Call, ${ }^{148}$ B. Caraway, ${ }^{148}$ J. Dittmann, ${ }^{148}$ K. Hatakeyama, ${ }^{148}$ C. Madrid, ${ }^{148}$ B. McMaster, ${ }^{148}$ N. Pastika, ${ }^{148}$ C. Smith,${ }^{148}$ R. Bartek, ${ }^{149}$ A. Dominguez, ${ }^{149}$ R. Uniyal, ${ }^{149}$ A. M. Vargas Hernandez, ${ }^{149}$ A. Buccilli, ${ }^{150}$ O. Charaf, ${ }^{150}$ S. I. Cooper ${ }^{150}$ S. V. Gleyzer, ${ }^{150}$ C. Henderson, ${ }^{150}$ P. Rumerio, ${ }^{150}$ C. West ${ }^{150}$ A. Akpinar, ${ }^{151}$ A. Albert, ${ }^{151}$ D. Arcaro, ${ }^{151}$ C. Cosby, ${ }^{151}$ Z. Demiragli, ${ }_{151}$ D. Gastler, ${ }^{151}$ C. Richardson, ${ }^{151}$ J. Rohlf, ${ }^{151}$ K. Salyer, ${ }^{151}$ D. Sperka, ${ }^{151}$ D. Spitzbart, ${ }^{151}$ I. Suarez,${ }^{151}$ S. Yuan, ${ }^{151}$ D. Zou, ${ }^{151}$ G. Benelli, ${ }^{152}$ B. Burkle, ${ }^{152}$ X. Coubez, ${ }^{152, t}$ D. Cutts, ${ }^{152}$ Y. t. Duh, ${ }^{152}$ M. Hadley, ${ }^{152}$ U. Heintz, ${ }^{152}$ J. M. Hogan, ${ }^{152, \text { bbbb }}$ K. H. M. Kwok, ${ }^{152}$ E. Laird, ${ }^{152}$ G. Landsberg, ${ }^{152}$ K. T. Lau, ${ }^{152}$ J. Lee, ${ }^{152}$ M. Narain, ${ }^{152}$ S. Sagir,${ }^{152, \text { cccc }}$ R. Syarif, ${ }^{152}$ E. Usai, ${ }^{152}$ W. Y. Wong,${ }^{152}$ D. Yu, ${ }^{152}$ W. Zhang, ${ }^{152}$ R. Band, ${ }^{153}$ C. Brainerd, ${ }^{153}$ R. Breedon, ${ }^{153}$ M. Calderon De La Barca Sanchez, ${ }^{153}$ M. Chertok, ${ }^{153}$ J. Conway, ${ }^{153}$ R. Conway, ${ }^{153}$ P. T. Cox, ${ }^{153}$ R. Erbacher, ${ }^{153}$ C. Flores,${ }^{153}$ G. Funk,${ }^{153}$ F. Jensen, ${ }^{153}$ W. Ko, ${ }^{153, a}$ O. Kukral, ${ }^{153}$ R. Lander, ${ }^{153}$ M. Mulhearn, ${ }^{153}$ D. Pellett, ${ }^{153}$ J. Pilot, ${ }^{153}$ M. Shi, ${ }^{153}$ D. Taylor, ${ }^{153}$ K. Tos, ${ }^{153}$ M. Tripathi, ${ }^{153}$ Y. Yao, ${ }^{153}$ F. Zhang, ${ }^{153}$ M. Bachtis, ${ }^{154}$ C. Bravo, ${ }^{154}$ R. Cousins, ${ }^{154}$ A. Dasgupta, ${ }^{154}$ A. Florent, ${ }^{154}$ D. Hamilton, ${ }^{154}$ J. Hauser, ${ }^{154}$ M. Ignatenko, ${ }^{154}$ T. Lam, ${ }^{154}$ N. Mccoll, ${ }^{154}$ W. A. Nash, ${ }^{154}$ S. Regnard, ${ }^{154}$ D. Saltzberg, ${ }^{154}$ C. Schnaible, ${ }^{154}$ B. Stone, ${ }^{154}$ V. Valuev, ${ }^{154}$ K. Burt, ${ }^{155}$ Y. Chen ${ }^{155}$ R. Clare, ${ }^{155}$ J. W. Gary, ${ }^{155}$ S. M. A. Ghiasi Shirazi, ${ }^{155}$ G. Hanson, ${ }^{155}$ G. Karapostoli, ${ }^{155}$ O. R. Long, ${ }^{155}$ N. Manganelli, ${ }^{155}$ M. Olmedo Negrete, ${ }^{155}$ M. I. Paneva, ${ }^{155}$ W. Si, ${ }^{155}$ S. Wimpenny, ${ }^{155}$ Y. Zhang, ${ }^{155}$ J. G. Branson, ${ }^{156}$ P. Chang, ${ }^{156}$ S. Cittolin, ${ }^{156}$ S. Cooperstein, ${ }^{156}$ N. Deelen, ${ }^{156}$ M. Derdzinski, ${ }^{156}$ J. Duarte, ${ }^{156}$ R. Gerosa, ${ }^{156}$ D. Gilbert,${ }^{156}$ B. Hashemi, ${ }^{156}$ D. Klein, ${ }^{156}$ V. Krutelyov, ${ }^{156}$ J. Letts, ${ }^{156}$ M. Masciovecchio, ${ }^{156}$ S. May, ${ }^{156}$ S. Padhi, ${ }^{156}$ M. Pieri, ${ }^{156}$ V. Sharma ${ }^{156}$ M. Tadel,${ }^{156}$ F. Würthwein, ${ }^{156}$ A. Yagil,${ }^{156}$ N. Amin, ${ }^{157}$ R. Bhandari, ${ }^{157}$ C. Campagnari, ${ }^{157}$ M. Citron, ${ }^{157}$ A. Dorsett, ${ }^{157}$ V. Dutta, ${ }^{157}$ J. Incandela, ${ }^{157}$ B. Marsh, ${ }^{157}$ H. Mei, ${ }^{157}$ A. Ovcharova, ${ }^{157}$ H. Qu, ${ }^{157}$ M. Quinnan, ${ }^{157}$ J. Richman, ${ }^{157}$ U. Sarica, ${ }^{157}$ D. Stuart, ${ }^{157}$ S. Wang, ${ }^{157}$ D. Anderson, ${ }^{158}$ A. Bornheim, ${ }^{158}$ O. Cerri, ${ }^{158}$ I. Dutta, ${ }^{158}$ J. M. Lawhorn, ${ }^{158}$ N. Lu, ${ }^{158}$ 
J. Mao, ${ }^{158}$ H. B. Newman, ${ }^{158}$ T. Q. Nguyen, ${ }^{158}$ J. Pata, ${ }^{158}$ M. Spiropulu, ${ }^{158}$ J. R. Vlimant, ${ }^{158}$ S. Xie, ${ }^{158}$ Z. Zhang, ${ }^{158}$ R. Y. Zhu, ${ }^{158}$ J. Alison, ${ }^{159}$ M. B. Andrews, ${ }^{159}$ T. Ferguson, ${ }^{159}$ T. Mudholkar, ${ }^{159}$ M. Paulini, ${ }^{159}$ M. Sun, ${ }^{159}$ I. Vorobiev, ${ }^{159}$ M. Weinberg, ${ }^{159}$ J. P. Cumalat, ${ }^{160}$ W. T. Ford, ${ }^{160}$ E. MacDonald, ${ }^{160}$ T. Mulholland, ${ }^{160}$ R. Patel,,${ }^{160}$ A. Perloff,,${ }^{160}$ K. Stenson, ${ }^{160}$ K. A. Ulmer, ${ }^{160}$ S. R. Wagner, ${ }^{160}$ J. Alexander, ${ }^{161}$ Y. Cheng, ${ }^{161}$ J. Chu, ${ }^{161}$ D. J. Cranshaw, ${ }^{161}$ A. Datta, ${ }^{161}$ A. Frankenthal ${ }^{161}$ K. Mcdermott, ${ }^{161}$ J. Monroy, ${ }^{161}$ J. R. Patterson, ${ }^{161}$ D. Quach, ${ }^{161}$ A. Ryd,${ }^{161}$ W. Sun, ${ }^{161}$ S. M. Tan, ${ }^{161}$ Z. Tao, ${ }^{161}$ J. Thom, ${ }^{161}$ P. Wittich, ${ }^{161}$ M. Zientek, ${ }^{161}$ S. Abdullin, ${ }^{162}$ M. Albrow ${ }^{162}$ M. Alyari, ${ }^{162}$ G. Apollinari, ${ }^{162}$ A. Apresyan, ${ }^{162}$ A. Apyan, ${ }^{162}$ S. Banerjee, ${ }^{162}$ L. A. T. Bauerdick, ${ }^{162}$ A. Beretvas, ${ }^{162}$ D. Berry, ${ }^{162}$ J. Berryhill, ${ }^{162}$ P. C. Bhat, ${ }^{162}$ K. Burkett, ${ }^{162}$ J. N. Butler, ${ }^{162}$ A. Canepa, ${ }^{162}$ G. B. Cerati, ${ }^{162}$ H. W. K. Cheung, ${ }^{162}$ F. Chlebana, ${ }^{162}$ M. Cremonesi, ${ }^{162}$ V. D. Elvira ${ }^{162}$ J. Freeman, ${ }^{162}$ Z. Gecse ${ }^{162}$ E. Gottschalk, ${ }^{162}$ L. Gray, ${ }^{162}$ D. Green,${ }^{162}$ S. Grünendahl,${ }^{162}$ O. Gutsche ${ }^{162}$ R. M. Harris ${ }^{162}$ S. Hasegawa, ${ }^{162}$ R. Heller ${ }^{162}$ T. C. Herwig, ${ }^{162}$ J. Hirschauer,${ }^{162}$ B. Jayatilaka, ${ }^{162}$ S. Jindariani, ${ }^{162}$ M. Johnson, ${ }^{162}$ U. Joshi, ${ }^{162}$ T. Klijnsma, ${ }^{162}$ B. Klima, ${ }^{162}$ M. J. Kortelainen, ${ }^{162}$ S. Lammel, ${ }^{162}$ J. Lewis, ${ }^{162}$ D. Lincoln, ${ }^{162}$ R. Lipton, ${ }^{162}$ M. Liu, ${ }^{162}$ T. Liu, ${ }^{162}$ J. Lykken, ${ }^{162}$ K. Maeshima, ${ }^{162}$ D. Mason, ${ }^{162}$ P. McBride, ${ }^{162}$ P. Merkel, ${ }^{162}$ S. Mrenna, ${ }^{162}$ S. Nahn, ${ }^{162}$ V. O’Dell, ${ }^{162}$ V. Papadimitriou, ${ }^{162}$ K. Pedro, ${ }^{162}$ C. Pena,${ }^{162, d d d d}$ O. Prokofyev, ${ }^{162}$ F. Ravera, ${ }^{162}$ A. Reinsvold Hall, ${ }^{162}$ L. Ristori, ${ }^{162}$ B. Schneider, ${ }^{162}$ E. Sexton-Kennedy, ${ }^{162}$ N. Smith, ${ }^{162}$ A. Soha, ${ }^{162}$ W. J. Spalding, ${ }^{162}$ L. Spiegel, ${ }^{162}$ S. Stoynev, ${ }^{162}$ J. Strait, ${ }^{162}$ L. Taylor, ${ }^{162}$ S. Tkaczyk, ${ }^{162}$ N. V. Tran, ${ }^{162}$ L. Uplegger, ${ }^{162}$ E. W. Vaandering, ${ }^{162}$ M. Wang, ${ }^{162}$ H. A. Weber, ${ }^{162}$ A. Woodard, ${ }^{162}$ D. Acosta, ${ }^{163}$ P. Avery, ${ }^{163}$ D. Bourilkov, ${ }^{163}$ L. Cadamuro, ${ }^{163}$ V. Cherepanov, ${ }^{163}$ F. Errico, ${ }^{163}$ R. D. Field, ${ }^{163}$ D. Guerrero, ${ }^{163}$ B. M. Joshi, ${ }^{163}$ M. Kim, ${ }^{163}$ J. Konigsberg, ${ }^{163}$ A. Korytov, ${ }^{163}$ K. H. Lo, ${ }^{163}$ K. Matchev ${ }^{163}$ N. Menendez, ${ }^{163}$ G. Mitselmakher,${ }^{163}$ D. Rosenzweig, ${ }^{163}$ K. Shi, ${ }^{163}$ J. Wang, ${ }^{163}$ S. Wang, ${ }^{163}$ X. Zuo, ${ }^{163}$ Y. R. Joshi, ${ }^{164}$ T. Adams, ${ }^{165}$ A. Askew, ${ }^{165}$ D. Diaz, ${ }^{165}$ R. Habibullah, ${ }^{165}$ S. Hagopian, ${ }^{165}$ V. Hagopian, ${ }^{165}$ K. F. Johnson, ${ }^{165}$ R. Khurana, ${ }^{165}$ T. Kolberg, ${ }^{165}$ G. Martinez, ${ }^{165}$ H. Prosper, ${ }^{165}$ C. Schiber ${ }^{165}$ R. Yohay ${ }^{165}$ J. Zhang, ${ }^{165}$ M. M. Baarmand, ${ }^{166}$ S. Butalla, ${ }^{166}$ T. Elkafrawy, ${ }^{166, \mathrm{~m}}$ M. Hohlmann, ${ }^{166}$ D. Noonan, ${ }^{166}$ M. Rahmani, ${ }^{166}$ M. Saunders, ${ }^{166}$ F. Yumiceva, ${ }^{166}$ M. R. Adams, ${ }^{167}$ L. Apanasevich, ${ }^{167}$ H. Becerril Gonzalez, ${ }^{167}$ R. Cavanaugh, ${ }^{167}$ X. Chen, ${ }^{167}$ S. Dittmer, ${ }^{167}$ O. Evdokimov, ${ }^{167}$ C. E. Gerber, ${ }^{167}$ D. A. Hangal, ${ }^{167}$ D. J. Hofman, ${ }^{167}$ C. Mills, ${ }^{167}$ G. Oh,${ }^{167}$ T. Roy, ${ }^{167}$ M. B. Tonjes,${ }^{167}$ N. Varelas, ${ }^{167}$ J. Viinikainen, ${ }^{167}$ H. Wang, ${ }^{167} \mathrm{X}$. Wang, ${ }^{167} \mathrm{Z}$. Wu, ${ }^{167}$ M. Alhusseini, ${ }^{168}$ B. Bilki, ${ }^{168, j \mathrm{jj}} \mathrm{K}$. Dilsiz,${ }^{168, \text { eeee }}$ S. Durgut, ${ }^{168}$ R. P. Gandrajula, ${ }^{168}$ M. Haytmyradov, ${ }^{168}$ V. Khristenko, ${ }^{168}$ O. K. Köseyan, ${ }^{168}$ J.-P. Merlo, ${ }^{168}$ A. Mestvirishvili, ${ }^{168, f f f f}$ A. Moeller, ${ }^{168}$ J. Nachtman, ${ }^{168}$ H. Ogul, ${ }^{168, g g g g}$ Y. Onel, ${ }^{168}$ F. Ozok,${ }^{168, \text { hhhh }}$ A. Penzo, ${ }^{168}$ C. Snyder,${ }^{168}$ E. Tiras, ${ }^{168}$ J. Wetzel, ${ }^{168}$ K. Yi, ${ }^{168, \text { iiii }}$ O. Amram, ${ }^{169}$ B. Blumenfeld, ${ }^{169}$ L. Corcodilos, ${ }^{169}$ M. Eminizer, ${ }^{169}$ A. V. Gritsan, ${ }^{169}$ S. Kyriacou, ${ }^{169}$ P. Maksimovic, ${ }^{169}$ C. Mantilla, ${ }^{169}$ J. Roskes, ${ }^{169}$ M. Swartz, ${ }^{169}$ T. Á. Vámi, ${ }^{169}$ C. Baldenegro Barrera ${ }^{170}$ P. Baringer,${ }^{170}$ A. Bean, ${ }^{170}$ A. Bylinkin, ${ }^{170}$ T. Isidori, ${ }^{170}$ S. Khalil, ${ }^{170}$ J. King, ${ }^{170}$ G. Krintiras, ${ }^{170}$ A. Kropivnitskaya, ${ }^{170}$ C. Lindsey, ${ }^{170}$ W. Mcbrayer, ${ }^{170}$ N. Minafra, ${ }^{170}$ M. Murray, ${ }^{170}$ C. Rogan, ${ }^{170}$ C. Royon, ${ }^{170}$ S. Sanders,${ }^{170}$ E. Schmitz, ${ }^{170}$ J. D. Tapia Takaki, ${ }^{170}$ Q. Wang, ${ }^{170}$ J. Williams,${ }^{170}$ G. Wilson, ${ }^{170}$ S. Duric, ${ }^{171}$ A. Ivanov, ${ }^{171}$ K. Kaadze,${ }^{171}$ D. Kim, ${ }^{171}$ Y. Maravin, ${ }^{171}$

D. R. Mendis ${ }^{171}$ T. Mitchell, ${ }^{171}$ A. Modak,${ }^{171}$ A. Mohammadi, ${ }^{171}$ F. Rebassoo, ${ }^{172}$ D. Wright, ${ }^{172}$ E. Adams,${ }^{173}$ A. Baden, ${ }^{173}$

O. Baron, ${ }^{173}$ A. Belloni, ${ }^{173}$ S. C. Eno, ${ }^{173}$ Y. Feng, ${ }^{173}$ N. J. Hadley, ${ }^{173}$ S. Jabeen, ${ }^{173}$ G. Y. Jeng, ${ }^{173}$ R. G. Kellogg, ${ }^{173}$

T. Koeth, ${ }^{173}$ A. C. Mignerey, ${ }^{173}$ S. Nabili, ${ }^{173}$ M. Seidel, ${ }^{173}$ A. Skuja, ${ }^{173}$ S. C. Tonwar, ${ }^{173}$ L. Wang, ${ }^{173}$ K. Wong, ${ }^{173}$ D. Abercrombie, ${ }^{174}$ B. Allen, ${ }^{174}$ R. Bi ${ }^{174}$ S. Brandt, ${ }^{174}$ W. Busza, ${ }^{174}$ I. A. Cali, ${ }^{174}$ Y. Chen,,${ }^{174}$ M. D'Alfonso, ${ }^{174}$ G. Gomez Ceballos, ${ }^{174}$ M. Goncharov, ${ }^{174}$ P. Harris, ${ }^{174}$ D. Hsu, ${ }^{174}$ M. Hu, ${ }^{174}$ M. Klute, ${ }^{174}$ D. Kovalskyi, ${ }^{174}$ J. Krupa, ${ }^{174}$ Y.-J. Lee, ${ }^{174}$ P. D. Luckey, ${ }^{174}$ B. Maier, ${ }^{174}$ A. C. Marini, ${ }^{174}$ C. Mcginn,,${ }^{174}$ C. Mironov, ${ }^{174}$ S. Narayanan, ${ }^{174}$ X. Niu, ${ }^{174}$ C. Paus, ${ }^{174}$ D. Rankin, ${ }^{174}$ C. Roland,${ }^{174}$ G. Roland, ${ }^{174}$ Z. Shi, ${ }^{174}$ G. S. F. Stephans,${ }^{174}$ K. Sumorok,${ }^{174}$ K. Tatar, ${ }^{174}$ D. Velicanu, ${ }^{174}$ J. Wang, ${ }^{174}$ T. W. Wang, ${ }^{174}$ Z. Wang, ${ }^{174}$ B. Wyslouch, ${ }^{174}$ R. M. Chatterjee, ${ }^{175}$ A. Evans, ${ }^{175}$ S. Guts, ${ }^{175, a}$ P. Hansen, ${ }^{175}$ J. Hiltbrand, ${ }^{175}$ Sh. Jain, ${ }^{175}$ M. Krohn,,${ }^{175}$ Y. Kubota, ${ }^{175}$ Z. Lesko, ${ }^{175}$ J. Mans, ${ }^{175}$ M. Revering, ${ }^{175}$ R. Rusack, ${ }^{175}$ R. Saradhy, ${ }^{175}$ N. Schroeder, ${ }^{175}$ N. Strobbe,${ }^{175}$ M. A. Wadud, ${ }^{175}$ J. G. Acosta, ${ }^{176}$ S. Oliveros, ${ }^{176}$ K. Bloom, ${ }^{177}$ S. Chauhan, ${ }^{177}$ D. R. Claes ${ }^{177}$ C. Fangmeier, ${ }^{177}$ L. Finco, ${ }^{177}$ F. Golf, ${ }^{177}$ J. R. González Fernández, ${ }^{177}$ I. Kravchenko, ${ }^{177}$ J. E. Siado, ${ }^{177}$ G. R. Snow ${ }^{177, a}$ B. Stieger, ${ }^{177}$ W. Tabb,${ }^{177}$ G. Agarwal, ${ }^{178}$ C. Harrington, ${ }^{178}$ I. Iashvili, ${ }^{178}$ A. Kharchilava, ${ }^{178}$ C. McLean, ${ }^{178}$ D. Nguyen, ${ }^{178}$ A. Parker, ${ }^{178}$ J. Pekkanen, ${ }^{178}$ S. Rappoccio, ${ }^{178}$ B. Roozbahani, ${ }^{178}$ G. Alverson, ${ }^{179}$ E. Barberis ${ }^{179}$ C. Freer, ${ }^{179}$ Y. Haddad ${ }^{179}$ A. Hortiangtham, ${ }^{179}$ G. Madigan, ${ }^{179}$ B. Marzocchi, ${ }^{179}$ D. M. Morse, ${ }^{179}$ V. Nguyen, ${ }^{179}$ T. Orimoto, ${ }^{179}$ L. Skinnari, ${ }^{179}$ A. Tishelman-Charny, ${ }^{179}$ T. Wamorkar, ${ }^{179}$ B. Wang, ${ }^{179}$ A. Wisecarver, ${ }^{179}$ D. Wood, ${ }^{179}$ S. Bhattacharya, ${ }^{180}$ J. Bueghly, ${ }^{180}$ Z. Chen, ${ }^{180}$ A. Gilbert, ${ }^{180}$ T. Gunter, ${ }^{180}$ K. A. Hahn, ${ }^{180}$ N. Odell ${ }^{180}$ M. H. Schmitt,${ }^{180}$ K. Sung, ${ }^{180}$ M. Velasco, ${ }^{180}$ R. Bucci, ${ }^{181}$ N. Dev,${ }^{181}$ R. Goldouzian, ${ }^{181}$ M. Hildreth,${ }^{181}$ K. Hurtado Anampa ${ }^{181}$ C. Jessop, ${ }^{181}$ D. J. Karmgard, ${ }^{181}$ K. Lannon, ${ }^{181}$ W. Li, ${ }^{181}$ N. Loukas, ${ }^{181}$ N. Marinelli, ${ }^{181}$ I. Mcalister, ${ }^{181}$ F. Meng, ${ }^{181}$ K. Mohrman, ${ }^{181}$ 
Y. Musienko, ${ }^{181, \text { rr }}$ R. Ruchti, ${ }^{181}$ P. Siddireddy, ${ }^{181}$ S. Taroni,${ }^{181}$ M. Wayne ${ }^{181}$ A. Wightman, ${ }^{181}$ M. Wolf, ${ }^{181}$ L. Zygala, ${ }^{181}$ J. Alimena, ${ }^{182}$ B. Bylsma, ${ }^{182}$ B. Cardwell, ${ }^{182}$ L. S. Durkin, ${ }^{182}$ B. Francis, ${ }^{182}$ C. Hill, ${ }^{182}$ W. Ji ${ }^{182}$ A. Lefeld, ${ }^{182}$ B. L. Winer, ${ }^{182}$ B. R. Yates ${ }^{182}$ G. Dezoort, ${ }^{183}$ P. Elmer, ${ }^{183}$ B. Greenberg, ${ }^{183}$ N. Haubrich, ${ }^{183}$ S. Higginbotham, ${ }^{183}$ A. Kalogeropoulos, ${ }^{183}$ G. Kopp, ${ }^{183}$ S. Kwan, ${ }^{183}$ D. Lange, ${ }^{183}$ M. T. Lucchini, ${ }^{183}$ J. Luo, ${ }^{183}$ D. Marlow, ${ }^{183}$ K. Mei, ${ }^{183}$ I. Ojalvo, ${ }^{183}$ J. Olsen, ${ }^{183}$ C. Palmer, ${ }^{183}$ P. Piroué, ${ }^{183}$ D. Stickland, ${ }^{183}$ C. Tully, ${ }^{183}$ S. Malik, ${ }^{184}$ S. Norberg,${ }^{184}$ V. E. Barnes, ${ }^{185}$ R. Chawla, ${ }^{185}$ S. Das, ${ }^{185}$ L. Gutay, ${ }^{185}$ M. Jones, ${ }^{185}$ A. W. Jung, ${ }^{185}$ B. Mahakud, ${ }^{185}$ G. Negro, ${ }^{185}$ N. Neumeister ${ }^{185}$ C. C. Peng, ${ }^{185}$ S. Piperov, ${ }^{185}$ H. Qiu, ${ }^{185}$ J. F. Schulte, ${ }^{185}$ N. Trevisani, ${ }^{185}$ F. Wang, ${ }^{185}$ R. Xiao, ${ }^{185}$ W. Xie,${ }^{185}$ T. Cheng, ${ }^{186}$ J. Dolen, ${ }^{186}$ N. Parashar, ${ }^{186}$ A. Baty, ${ }^{187}$ S. Dildick, ${ }^{187}$ K. M. Ecklund, ${ }^{187}$ S. Freed, ${ }^{187}$ F. J. M. Geurts, ${ }^{187}$ M. Kilpatrick, ${ }^{187}$ A. Kumar,${ }^{187}$ W. Li $1{ }^{187}$ B. P. Padley, ${ }^{187}$ R. Redjimi, ${ }^{187}$ J. Roberts, ${ }^{187, a}$ J. Rorie, ${ }^{187}$ W. Shi, ${ }^{187}$ A. G. Stahl Leiton, ${ }^{187}$ Z. Tu, ${ }^{187}$ A. Zhang, ${ }^{187}$ A. Bodek, ${ }^{188}$ P. de Barbaro, ${ }^{188}$ R. Demina, ${ }^{188}$ J. L. Dulemba, ${ }^{188}$ C. Fallon, ${ }^{188}$ T. Ferbel, ${ }^{188}$ M. Galanti, ${ }^{188}$

A. Garcia-Bellido, ${ }^{188}$ O. Hindrichs, ${ }^{188}$ A. Khukhunaishvili, ${ }^{188}$ E. Ranken, ${ }^{188}$ R. Taus, ${ }^{188}$ B. Chiarito, ${ }^{189}$ J. P. Chou, ${ }^{189}$ A. Gandrakota, ${ }^{189}$ Y. Gershtein, ${ }^{189}$ E. Halkiadakis, ${ }^{189}$ A. Hart, ${ }^{189}$ M. Heindl,${ }^{189}$ E. Hughes, ${ }^{189}$ S. Kaplan, ${ }^{189}$

O. Karacheban, ${ }^{189, w}$ I. Laflotte, ${ }^{189}$ A. Lath ${ }^{189}$ R. Montalvo, ${ }^{189}$ K. Nash, ${ }^{189}$ M. Osherson, ${ }^{189}$ S. Salur, ${ }^{189}$ S. Schnetzer, ${ }^{189}$ S. Somalwar, ${ }^{189}$ R. Stone, ${ }^{189}$ S. A. Thayil,${ }^{189}$ S. Thomas, ${ }^{189}$ H. Acharya, ${ }^{190}$ A. G. Delannoy, ${ }^{190}$ S. Spanier,${ }^{190}$

O. Bouhali, ${ }^{191, j \mathrm{jjj}}$ M. Dalchenko, ${ }^{191}$ A. Delgado, ${ }^{191}$ R. Eusebi, ${ }^{191}$ J. Gilmore, ${ }^{191}$ T. Huang, ${ }^{191}$ T. Kamon, ${ }^{191, \mathrm{kkkk}}$ H. Kim, ${ }^{191}$ S. Luo, ${ }^{191}$ S. Malhotra, ${ }^{191}$ D. Marley, ${ }^{191}$ R. Mueller, ${ }^{191}$ D. Overton, ${ }^{191}$ L. Perniè, ${ }^{191}$ D. Rathjens, ${ }^{191}$ A. Safonov, ${ }^{191}$ N. Akchurin, ${ }^{192}$ J. Damgov, ${ }^{192}$ V. Hegde, ${ }^{192}$ S. Kunori, ${ }^{192}$ K. Lamichhane, ${ }^{192}$ S. W. Lee, ${ }^{192}$ T. Mengke, ${ }^{192}$ S. Muthumuni, ${ }^{192}$ T. Peltola, ${ }^{192}$ S. Undleeb, ${ }^{192}$ I. Volobouev, ${ }^{192}$ Z. Wang, ${ }^{192}$ A. Whitbeck, ${ }^{192}$ E. Appelt, ${ }^{193}$ S. Greene, ${ }^{193}$ A. Gurrola, ${ }^{193}$ R. Janjam, ${ }^{193}$ W. Johns, ${ }^{193}$ C. Maguire, ${ }^{193}$ A. Melo, ${ }^{193}$ H. Ni,${ }^{193}$ K. Padeken, ${ }^{193}$ F. Romeo, ${ }^{193}$ P. Sheldon, ${ }^{193}$ S. Tuo, ${ }^{193}$ J. Velkovska, ${ }^{193}$ M. Verweij, ${ }^{193}$ L. Ang, ${ }^{194}$ M. W. Arenton, ${ }^{194}$ B. Cox,${ }^{194}$ G. Cummings,${ }^{194}$ J. Hakala, ${ }^{194}$ R. Hirosky, ${ }^{194}$ M. Joyce, ${ }^{194}$ A. Ledovskoy, ${ }^{194}$ C. Neu, ${ }^{194}$ B. Tannenwald, ${ }^{194}$ Y. Wang, ${ }^{194}$ E. Wolfe, ${ }^{194}$ F. Xia, ${ }^{194}$ P. E. Karchin, ${ }^{195}$ N. Poudyal, ${ }^{195}$ J. Sturdy, ${ }^{195}$ P. Thapa,${ }^{195}$ K. Black, ${ }^{196}$ T. Bose, ${ }^{196}$ J. Buchanan, ${ }^{196}$ C. Caillol,${ }^{196}$ S. Dasu, ${ }^{196}$ I. De Bruyn, ${ }^{196}$ L. Dodd, ${ }^{196}$ C. Galloni, ${ }^{196}$ H. He,${ }^{196}$ M. Herndon, ${ }^{196}$ A. Hervé, ${ }^{196}$ U. Hussain, ${ }^{196}$ A. Lanaro, ${ }^{196}$ A. Loeliger, ${ }^{196}$ R. Loveless, ${ }^{196}$ J. Madhusudanan Sreekala, ${ }^{196}$ A. Mallampalli, ${ }^{196}$ D. Pinna, ${ }^{196}$ T. Ruggles, ${ }^{196}$ A. Savin, ${ }^{196}$ V. Shang, ${ }^{196}$ V. Sharma, ${ }^{196}$ W. H. Smith, ${ }^{196}$ D. Teague, ${ }^{196}$ S. Trembath-reichert, ${ }^{196}$ and W. Vetens ${ }^{196}$

(CMS Collaboration)

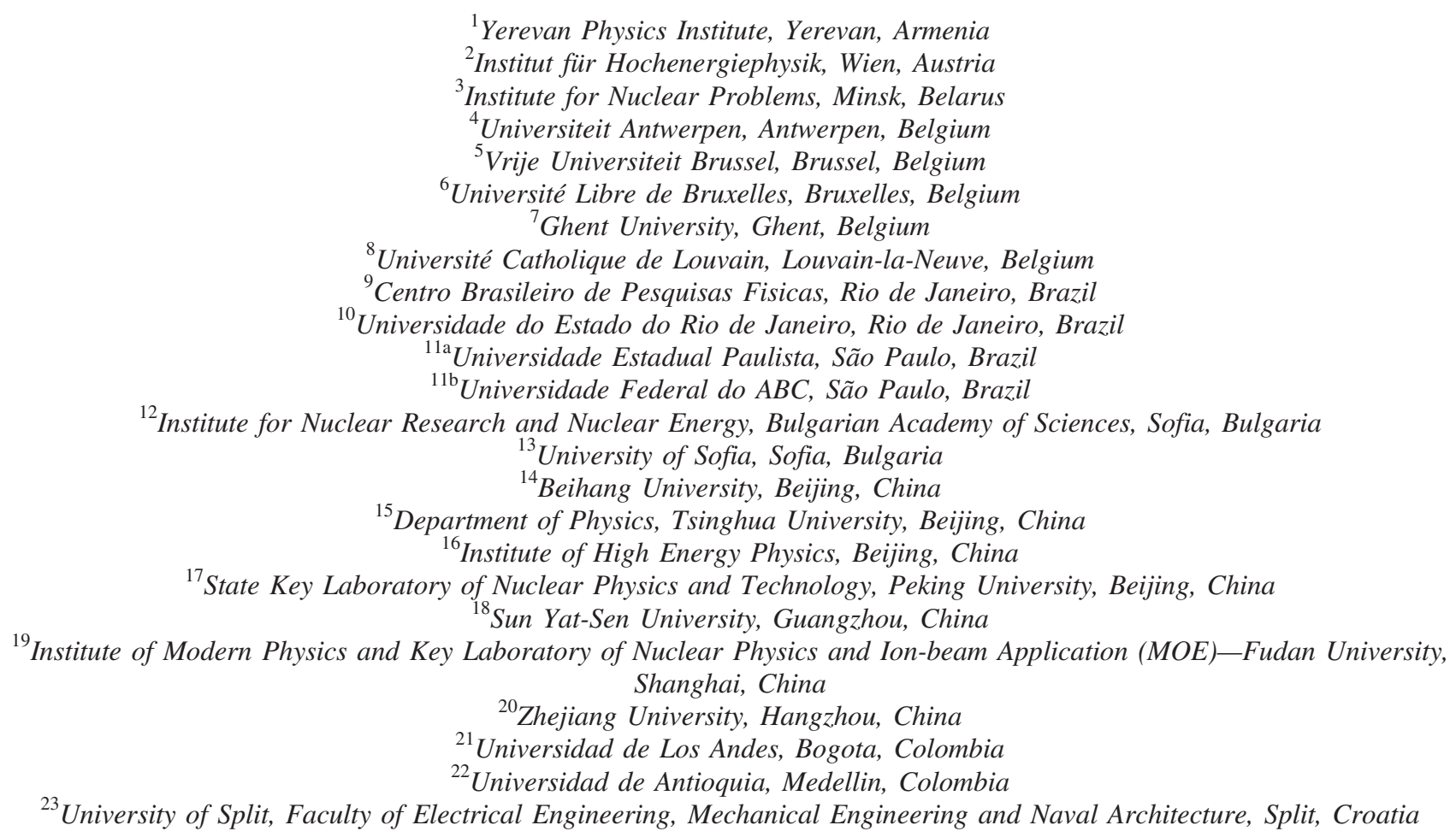


${ }^{24}$ University of Split, Faculty of Science, Split, Croatia

${ }^{25}$ Institute Rudjer Boskovic, Zagreb, Croatia

${ }^{26}$ University of Cyprus, Nicosia, Cyprus

${ }^{27}$ Charles University, Prague, Czech Republic

${ }^{28}$ Escuela Politecnica Nacional, Quito, Ecuador

${ }^{29}$ Universidad San Francisco de Quito, Quito, Ecuador

${ }^{30}$ Academy of Scientific Research and Technology of the Arab Republic of Egypt, Egyptian Network of High Energy Physics, Cairo, Egypt

${ }^{31}$ Center for High Energy Physics (CHEP-FU), Fayoum University, El-Fayoum, Egypt

${ }^{32}$ National Institute of Chemical Physics and Biophysics, Tallinn, Estonia

${ }^{33}$ Department of Physics, University of Helsinki, Helsinki, Finland

${ }^{34}$ Helsinki Institute of Physics, Helsinki, Finland

${ }^{35}$ Lappeenranta University of Technology, Lappeenranta, Finland

${ }^{36}$ IRFU, CEA, Université Paris-Saclay, Gif-sur-Yvette, France

${ }^{37}$ Laboratoire Leprince-Ringuet, CNRS/IN2P3, Ecole Polytechnique, Institut Polytechnique de Paris, Paris, France

${ }^{38}$ Université de Strasbourg, CNRS, IPHC UMR 7178, Strasbourg, France

${ }^{39}$ Université de Lyon, Université Claude Bernard Lyon 1, CNRS-IN2P3, Institut de Physique Nucléaire de Lyon, Villeurbanne, France

${ }^{40}$ Georgian Technical University, Tbilisi, Georgia

${ }^{41}$ Tbilisi State University, Tbilisi, Georgia

${ }^{42}$ RWTH Aachen University, I. Physikalisches Institut, Aachen, Germany

${ }^{43}$ RWTH Aachen University, III. Physikalisches Institut A, Aachen, Germany

${ }^{44}$ RWTH Aachen University, III. Physikalisches Institut B, Aachen, Germany

${ }^{45}$ Deutsches Elektronen-Synchrotron, Hamburg, Germany

${ }^{46}$ University of Hamburg, Hamburg, Germany

${ }^{47}$ Karlsruher Institut fuer Technologie, Karlsruhe, Germany

${ }^{48}$ Institute of Nuclear and Particle Physics (INPP), NCSR Demokritos, Aghia Paraskevi, Greece

${ }^{49}$ National and Kapodistrian University of Athens, Athens, Greece

${ }^{50}$ National Technical University of Athens, Athens, Greece

${ }^{51}$ University of Ioánnina, Ioánnina, Greece

${ }^{52}$ MTA-ELTE Lendület CMS Particle and Nuclear Physics Group, Eötvös Loránd University, Budapest, Hungary

${ }^{53}$ Wigner Research Centre for Physics, Budapest, Hungary

${ }^{54}$ Institute of Nuclear Research ATOMKI, Debrecen, Hungary

${ }^{55}$ Institute of Physics, University of Debrecen, Debrecen, Hungary

${ }^{56}$ Eszterhazy Karoly University, Karoly Robert Campus, Gyongyos, Hungary

${ }^{57}$ Indian Institute of Science (IISc), Bangalore, India

${ }^{58}$ National Institute of Science Education and Research, HBNI, Bhubaneswar, India

${ }^{59}$ Panjab University, Chandigarh, India

${ }^{60}$ University of Delhi, Delhi, India

${ }^{61}$ Saha Institute of Nuclear Physics, HBNI, Kolkata,India

${ }^{62}$ Indian Institute of Technology Madras, Madras, India

${ }^{63}$ Bhabha Atomic Research Centre, Mumbai, India

${ }^{64}$ Tata Institute of Fundamental Research-A, Mumbai, India

${ }^{65}$ Tata Institute of Fundamental Research-B, Mumbai, India

${ }^{66}$ Indian Institute of Science Education and Research (IISER), Pune, India

${ }^{67}$ Department of Physics, Isfahan University of Technology, Isfahan, Iran

${ }^{68}$ Institute for Research in Fundamental Sciences (IPM), Tehran, Iran

${ }^{69}$ University College Dublin, Dublin, Ireland

${ }^{70 a}$ INFN Sezione di Bari

${ }^{70 \mathrm{~b}}$ Università di Bari

${ }^{70 \mathrm{c}}$ Politecnico di Bari

${ }^{71 a}$ INFN Sezione di Bologna, Bologna, Italy

${ }^{71 \mathrm{~b}}$ Università di Bologna, Bologna, Italy

${ }^{72 a}$ INFN Sezione di Catania, Catania, Italy

${ }^{72 \mathrm{~b}}$ Università di Catania, Catania, Italy

${ }^{73}$ INFN Sezione di Firenze, Firenze, Italy

${ }^{73 \mathrm{~b}}$ Università di Firenze, Firenze, Italy

${ }^{74}$ INFN Laboratori Nazionali di Frascati, Frascati, Italy

${ }^{75 a}$ INFN Sezione di Genova, Genova, Italy

${ }^{75 b}$ Università di Genova, Genova, Italy

${ }^{76 a}$ INFN Sezione di Milano-Bicocca, Milano, Italy 
${ }^{76 \mathrm{~b}}$ Università di Milano-Bicocca, Milano, Italy

${ }^{77 a}$ INFN Sezione di Napoli, Napoli, Italy

${ }^{77 b}$ Università di Napoli 'Federico II', Napoli, Italy

${ }^{77 \mathrm{c}}$ Università della Basilicata, Potenza, Italy

${ }^{77 \mathrm{~d}}$ Università G. Marconi, Roma, Italy

${ }^{78 a}$ INFN Sezione di Padova, Padova, Italy

${ }^{78 b}$ Università di Padova, Padova, Italy

${ }^{78 \mathrm{c}}$ Università di Trento, Trento, Italy

${ }^{79 a}$ INFN Sezione di Pavia

${ }^{79 b}$ Università di Pavia

${ }^{80 a}$ INFN Sezione di Perugia, Perugia, Italy

${ }^{80 \mathrm{~b}}$ Università di Perugia, Perugia, Italy

${ }^{81 a}$ INFN Sezione di Pisa, Pisa, Italy

${ }^{81 \mathrm{~b}}$ Università di Pisa, Pisa, Italy

${ }^{81 \mathrm{c}}$ Scuola Normale Superiore di Pisa, Pisa, Italy

${ }^{82 a}$ INFN Sezione di Roma, Rome, Italy

${ }^{82 \mathrm{~b}}$ Sapienza Università di Roma, Rome, Italy

${ }^{83 a}$ INFN Sezione di Torino, Torino, Italy

${ }^{83 \mathrm{~b}}$ Università di Torino, Torino, Italy

${ }^{83 \mathrm{c}}$ Università del Piemonte Orientale, Novara, Italy

${ }^{84 a}$ INFN Sezione di Trieste, Trieste, Italy

${ }^{84 \mathrm{~b}}$ Università di Trieste, Trieste, Italy

${ }^{85}$ Kyungpook National University, Daegu, Korea

${ }^{86}$ Chonnam National University, Institute for Universe and Elementary Particles, Kwangju, Korea

${ }^{87}$ Hanyang University, Seoul, Korea

${ }^{88}$ Korea University, Seoul, Korea

${ }^{89}$ Kyung Hee University, Department of Physics, Seoul, Republic of Korea

${ }^{90}$ Sejong University, Seoul, Korea

${ }^{91}$ Seoul National University, Seoul, Korea

${ }^{92}$ University of Seoul, Seoul, Korea

${ }^{93}$ Yonsei University, Department of Physics, Seoul, Korea

${ }^{94}$ Sungkyunkwan University, Suwon, Korea

${ }^{95}$ Riga Technical University, Riga, Latvia

${ }^{96}$ Vilnius University, Vilnius, Lithuania

${ }^{97}$ National Centre for Particle Physics, Universiti Malaya, Kuala Lumpur, Malaysia

${ }^{98}$ Universidad de Sonora (UNISON), Hermosillo, Mexico

${ }^{99}$ Centro de Investigacion y de Estudios Avanzados del IPN, Mexico City, Mexico

${ }^{100}$ Universidad Iberoamericana, Mexico City, Mexico

${ }^{101}$ Benemerita Universidad Autonoma de Puebla, Puebla, Mexico

${ }^{102}$ Universidad Autónoma de San Luis Potosí, San Luis Potosí, Mexico

${ }^{103}$ University of Montenegro, Podgorica, Montenegro

${ }^{104}$ University of Auckland, Auckland, New Zealand

${ }^{105}$ University of Canterbury, Christchurch, New Zealand

${ }^{106}$ National Centre for Physics, Quaid-I-Azam University, Islamabad, Pakistan

${ }^{107}$ AGH University of Science and Technology Faculty of Computer Science, Electronics and Telecommunications, Krakow, Poland

${ }^{108}$ National Centre for Nuclear Research, Swierk, Poland

${ }^{109}$ Institute of Experimental Physics, Faculty of Physics, University of Warsaw, Warsaw, Poland

${ }^{110}$ Laboratório de Instrumentação e Física Experimental de Partículas, Lisboa, Portugal

${ }^{111}$ Joint Institute for Nuclear Research, Dubna, Russia

${ }^{112}$ Petersburg Nuclear Physics Institute, Gatchina (St. Petersburg), Russia

${ }^{113}$ Institute for Nuclear Research, Moscow, Russia

${ }^{114}$ Institute for Theoretical and Experimental Physics named by A.I. Alikhanov of NRC 'Kurchatov Institute', Moscow, Russia

${ }^{115}$ Moscow Institute of Physics and Technology, Moscow, Russia

${ }^{116}$ National Research Nuclear University 'Moscow Engineering Physics Institute' (MEPhI), Moscow, Russia

${ }^{117}$ P.N. Lebedev Physical Institute, Moscow, Russia

${ }^{118}$ Skobeltsyn Institute of Nuclear Physics, Lomonosov Moscow State University, Moscow, Russia

${ }^{119}$ Novosibirsk State University (NSU), Novosibirsk, Russia

${ }^{120}$ Institute for High Energy Physics of National Research Centre 'Kurchatov Institute', Protvino, Russia

${ }^{121}$ National Research Tomsk Polytechnic University, Tomsk, Russia

${ }^{122}$ Tomsk State University, Tomsk, Russia 
${ }^{23}$ University of Belgrade: Faculty of Physics and VINCA Institute of Nuclear Sciences, Belgrade, Serbia

${ }^{124}$ Centro de Investigaciones Energéticas Medioambientales y Tecnológicas (CIEMAT), Madrid, Spain

${ }^{125}$ Universidad Autónoma de Madrid, Madrid, Spain

${ }^{126}$ Universidad de Oviedo, Instituto Universitario de Ciencias y Tecnologías Espaciales de Asturias (ICTEA), Oviedo, Spain

${ }^{127}$ Instituto de Física de Cantabria (IFCA), CSIC-Universidad de Cantabria, Santander, Spain

${ }^{128}$ University of Colombo, Colombo, Sri Lanka

${ }^{129}$ University of Ruhuna, Department of Physics, Matara, Sri Lanka

${ }^{130}$ CERN, European Organization for Nuclear Research, Geneva, Switzerland

${ }^{131}$ Paul Scherrer Institut, Villigen, Switzerland

${ }^{132}$ ETH Zurich-Institute for Particle Physics and Astrophysics (IPA), Zurich, Switzerland

${ }^{133}$ Universität Zürich, Zurich, Switzerland

${ }^{134}$ National Central University, Chung-Li, Taiwan

${ }^{135}$ National Taiwan University (NTU), Taipei, Taiwan

${ }^{136}$ Chulalongkorn University, Faculty of Science, Department of Physics, Bangkok, Thailand

${ }^{137}$ Çukurova University, Physics Department, Science and Art Faculty, Adana, Turkey

${ }^{138}$ Middle East Technical University, Physics Department, Ankara, Turkey

${ }^{139}$ Bogazici University, Istanbul, Turkey

${ }^{140}$ Istanbul Technical University, Istanbul, Turkey

${ }^{141}$ Istanbul University, Istanbul, Turkey

${ }^{142}$ Institute for Scintillation Materials of National Academy of Science of Ukraine, Kharkov, Ukraine

${ }^{143}$ National Scientific Center, Kharkov Institute of Physics and Technology, Kharkov, Ukraine

${ }^{144}$ University of Bristol, Bristol, United Kingdom

${ }^{145}$ Rutherford Appleton Laboratory, Didcot, United Kingdom

${ }^{146}$ Imperial College, London, United Kingdom

${ }^{147}$ Brunel University, Uxbridge, United Kingdom

${ }^{148}$ Baylor University, Waco, Texas, USA

${ }^{149}$ Catholic University of America, Washington, DC, USA

${ }^{150}$ The University of Alabama, Tuscaloosa, Alabama, USA

${ }^{151}$ Boston University, Boston, Massachusetts, USA

${ }^{152}$ Brown University, Providence, Rhode Island, USA

${ }^{153}$ University of California, Davis, Davis, California, USA

${ }^{154}$ University of California, Los Angeles, California, USA

${ }^{155}$ University of California, Riverside, Riverside, California, USA

${ }^{156}$ University of California, San Diego, La Jolla, California, USA

${ }^{157}$ University of California, Santa Barbara-Department of Physics, Santa Barbara, California, USA

${ }^{158}$ California Institute of Technology, Pasadena, California, USA

${ }^{159}$ Carnegie Mellon University, Pittsburgh, Pennsylvania, USA

${ }^{160}$ University of Colorado Boulder, Boulder, Colorado, USA

${ }^{161}$ Cornell University, Ithaca, New York, USA

${ }^{162}$ Fermi National Accelerator Laboratory, Batavia, Illinois, USA

${ }^{163}$ University of Florida, Gainesville, Florida, USA

${ }^{164}$ Florida International University, Miami, Florida, USA

${ }^{165}$ Florida State University, Tallahassee, Florida, USA

${ }^{166}$ Florida Institute of Technology, Melbourne, Florida, USA

${ }^{167}$ University of Illinois at Chicago (UIC), Chicago, Illinois, USA

${ }^{168}$ The University of Iowa, Iowa City, Iowa, USA

${ }^{169}$ Johns Hopkins University, Baltimore, Maryland, USA

${ }^{170}$ The University of Kansas, Lawrence, Kansas, USA

${ }^{171}$ Kansas State University, Manhattan, Kansas, USA

${ }^{172}$ Lawrence Livermore National Laboratory, Livermore, California, USA

${ }^{173}$ University of Maryland, College Park, Maryland, USA

${ }^{174}$ Massachusetts Institute of Technology, Cambridge, Massachusetts, USA

${ }^{175}$ University of Minnesota, Minneapolis, Minnesota, USA

${ }^{176}$ University of Mississippi, Oxford, Mississippi, USA

${ }^{177}$ University of Nebraska-Lincoln, Lincoln, Nebraska, USA

${ }^{178}$ State University of New York at Buffalo, Buffalo, New York, USA

${ }^{179}$ Northeastern University, Boston, Massachusetts, USA

${ }^{180}$ Northwestern University, Evanston, Illinois, USA

${ }^{181}$ University of Notre Dame, Notre Dame, Indiana, USA

${ }^{182}$ The Ohio State University, Columbus, Ohio, USA 


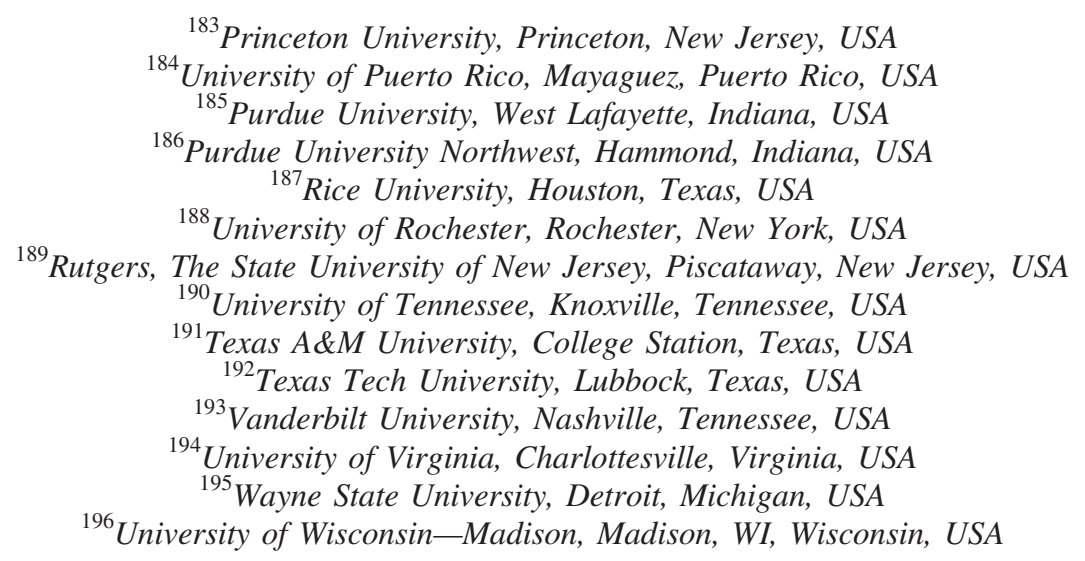

${ }^{\mathrm{a}}$ Deceased.

${ }^{\mathrm{b}}$ Also at Vienna University of Technology, Vienna, Austria.

${ }^{\mathrm{c}}$ Also at Department of Basic and Applied Sciences, Faculty of Engineering, Arab Academy for Science, Technology and Maritime Transport.

${ }^{\mathrm{d}}$ Also at Université Libre de Bruxelles, Bruxelles, Belgium.

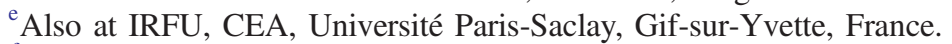

${ }^{\mathrm{f}}$ Also at Universidade Estadual de Campinas, Campinas, Brazil.

${ }^{g}$ Also at Federal University of Rio Grande do Sul, Porto Alegre, Brazil.

${ }^{\mathrm{h}}$ Also at UFMS.

${ }^{\mathrm{i}}$ Also at Universidade Federal de Pelotas, Pelotas, Brazil.

${ }^{\mathrm{j}}$ Also at University of Chinese Academy of Sciences.

kAlso at Institute for Theoretical and Experimental Physics named by A.I. Alikhanov of NRC 'Kurchatov Institute', Moscow, Russia.

${ }^{1}$ Also at Joint Institute for Nuclear Research, Dubna, Russia.

${ }^{\mathrm{m}}$ Also at Ain Shams University, Cairo, Egypt.

${ }^{\mathrm{n}}$ Also at British University in Egypt, Cairo, Egypt.

${ }^{\circ}$ Also at Fayoum University, El-Fayoum, Egypt.

${ }^{\mathrm{p}}$ Also at Purdue University, West Lafayette, Indiana, USA.

${ }^{\mathrm{q}}$ Also at Université de Haute Alsace, Mulhouse, France.

${ }^{\mathrm{r}}$ Also at Erzincan Binali Yildirim University, Erzincan, Turkey.

${ }^{\mathrm{s}}$ Also at CERN, European Organization for Nuclear Research, Geneva, Switzerland.

${ }^{\mathrm{t}}$ Also at RWTH Aachen University, III. Physikalisches Institut A, Aachen, Germany.

${ }^{\mathrm{A}}$ Also at University of Hamburg, Hamburg, Germany.

${ }^{v}$ Also at Department of Physics, Isfahan University of Technology, Isfahan, Iran.

${ }^{\mathrm{w}}$ Also at Brandenburg University of Technology, Cottbus, Germany.

${ }^{x}$ Also at Skobeltsyn Institute of Nuclear Physics, Lomonosov Moscow State University, Moscow, Russia.

${ }^{y}$ Also at Institute of Physics, University of Debrecen, Debrecen, Hungary.

${ }^{\mathrm{z}}$ Also at Physics Department, Faculty of Science, Assiut University.

${ }^{\text {aa }}$ Also at Institute of Nuclear Research ATOMKI, Debrecen, Hungary.

${ }^{\mathrm{bb}}$ Also at MTA-ELTE Lendület CMS Particle and Nuclear Physics Group, Eötvös Loránd University, Budapest, Hungary.

${ }^{\mathrm{cc}}$ Also at IIT Bhubaneswar, Bhubaneswar, India.

${ }^{\mathrm{dd}}$ Also at Institute of Physics, Bhubaneswar, India.

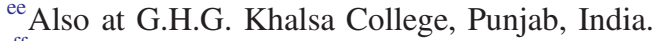

${ }^{\mathrm{ff}}$ Also at Shoolini University, Solan, India.

${ }^{\mathrm{gg}}$ Also at University of Hyderabad, Hyderabad, India.

${ }^{\text {hh }}$ Also at University of Visva-Bharati, Santiniketan, India.

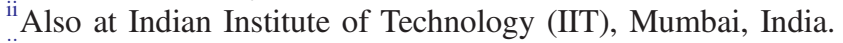

${ }^{\mathrm{jj}}$ Also at Deutsches Elektronen-Synchrotron, Hamburg, Germany.

${ }^{\mathrm{kk}}$ Also at Department of Physics, University of Science and Technology of Mazandaran, Behshahr, Iran.

${ }^{11}$ Also at INFN Sezione di Bari, Università di Bari, Politecnico di Bari, Bari, Italy.

${ }^{\mathrm{mm}}$ Also at Italian National Agency for New Technologies, Energy and Sustainable Economic Development.

${ }^{n n}$ Also at Centro Siciliano di Fisica Nucleare e di Struttura Della Materia.

${ }^{00}$ Also at Riga Technical University, Riga, Latvia.

${ }^{\mathrm{pp}}$ Also at Consejo Nacional de Ciencia y Tecnología, Mexico City, Mexico.

${ }^{\mathrm{qq}}$ Also at Warsaw University of Technology, Institute of Electronic Systems, Warsaw, Poland.

${ }^{\mathrm{rr}}$ Also at Institute for Nuclear Research, Moscow, Russia. 


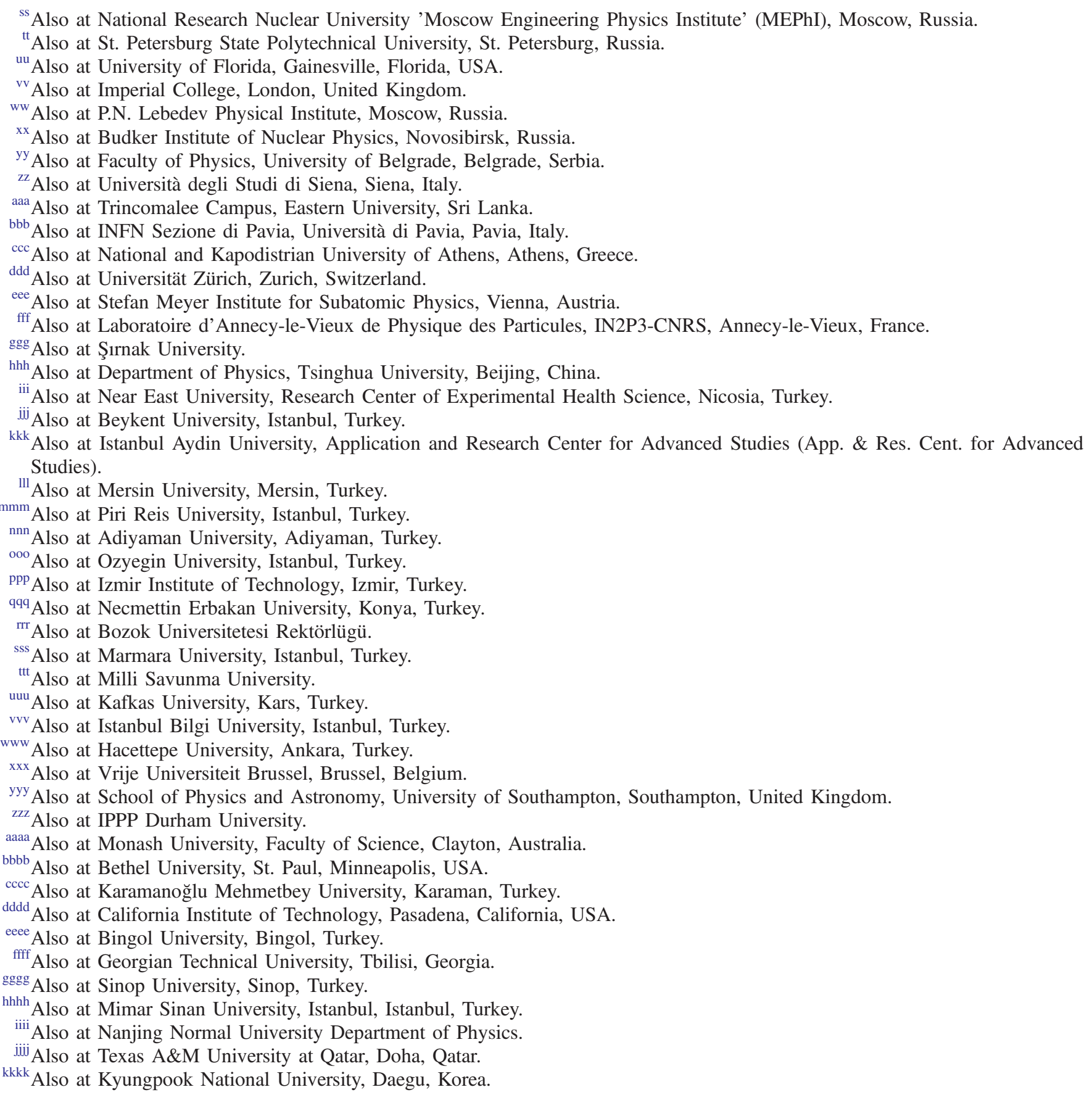

